\title{
Posterior Consistency for Bayesian Inverse Problems through Stability and Regression Results
}

\author{
Sebastian J. Vollmer 团 \\ Mathematics Institute, Zeeman Building, University of Warwick, Coventry CV4 7AL, \\ UK. \\ E-mail: Sebastian.Vollmer@stats.ox.ac.uk
}

\begin{abstract}
In the Bayesian approach, the a priori knowledge about the input of a mathematical model is described via a probability measure. The joint distribution of the unknown input and the data is then conditioned, using Bayes' formula, giving rise to the posterior distribution on the unknown input. In this setting we prove posterior consistency for nonlinear inverse problems: a sequence of data is considered, with diminishing fluctuations around a single truth and it is then of interest to show that the resulting sequence of posterior measures arising from this sequence of data concentrates around the truth used to generate the data. Posterior consistency justifies the use of the Bayesian approach very much in the same way as error bounds and convergence results for regularisation techniques do. As a guiding example, we consider the inverse problem of reconstructing the diffusion coefficient from noisy observations of the solution to an elliptic PDE in divergence form. This problem is approached by splitting the forward operator into the underlying continuum model and a simpler observation operator based on the output of the model.

In general, these splittings allow us to conclude posterior consistency provided a deterministic stability result for the underlying inverse problem and a posterior consistency result for the Bayesian regression problem with the push-forward prior.

Moreover, we prove posterior consistency for the Bayesian regression problem based on the regularity, the tail behaviour and the small ball probabilities of the prior.
\end{abstract}

AMS classification scheme numbers: 35R30, 62C10, 62G20

Submitted to: Inverse Problems

$\ddagger$ Present address: Department of Statistics, University of Oxford, 1 South Parks Road, Oxford OX1 3TG. 


\section{Introduction}

Many mathematical models used in science and technology contain parameters for which a direct observation is very difficult. A good example is subsurface geophysics. The aim in subsurface geophysics is the reconstruction of subsurface properties such as density and permeability given measurements on the surface.Using the laws of physics, these properties can be used as parameters of a forward model mapping them to the measurements which we subsequently call data.

Inverting such a relationship is non-trivial and lies in the focus of the area of inverse problems. Classically, these parameters are estimated by minimisation of a regularised least squares functional which is based on the data output mismatch (Tikhonov). The idea of this approach is to use optimisation techniques aiming at parameters that produce nearly the same noiseless output as the given noisy data while being not too irregular. However, it is difficult to quantify how the noise in the data translates into the uncertainty of the reconstructed parameters for this method. Uncertainty quantification is much more straightforward in the Bayesian approach. The basic idea of the Bayesian method is that not all parameter choices are a priori equally likely. Instead, the parameters are artificially treated as random variables by modelling their distribution using a priori knowledge. This distribution is accordingly called the prior. For a specific forward model and given the distribution of the observational noise, the parameters and the data can be treated as jointly varying random variables. Under mild conditions, the prior can then be updated by conditioning the parameters on the data.

The posterior is one of the main tools for making inference about the parameters. Possible estimates include approximation of the posterior mean or the maximum a posteriori (MAP) estimator. Moreover, it is possible to quantify the uncertainty of the reconstructed parameter by posterior variance or posterior probability of a set around for example an estimate of the parameters under consideration.

The main focus of this article lies on posterior consistency which quantifies the quality of the resulting posterior in a thought experiment. As for any evaluation for an approach to inverse problems, an identical twin experiment is performed, that is for a fixed set of parametersand artificial data is generated. It is conceivable to expect that, under appropriate conditions, the posterior concentrates around this set of 'true' parameters. Results of this type are called posterior consistency. It justifies the Bayesian method by establishing that this method recovers the 'true' parameters sometimes with a specific rate.

So far, there are only posterior consistency results available for linear forward models and mainly Gaussian priors [30, 1, 35, 20]. In this article, we prove posterior consistency of nonlinear inverse problems with explicit bounds on the rate. The main idea behind our posterior consistency results is to use stability properties of the deterministic inverse problem to reduce posterior consistency of a nonlinear inverse problem to posterior consistency of a Bayesian non-parametric regression problem. Our 
guiding example is the inverse problem of reconstructing the diffusion coefficient from measurements of the pressure. More precisely, we assume that the relation between the diffusion coefficient $a$ and the pressure $p$ satisfies the following partial differential equation (PDE) with Dirichlet boundary conditions

$$
\left\{\begin{array}{cl}
-\nabla \cdot(a \nabla p)=f(x) & \text { in } D \\
p=0 & \text { on } \partial D
\end{array}\right.
$$

where $D$ is a bounded smooth domain in $\mathbb{R}^{d}$. For this guiding example the required stability results are due to [36]. However, our methods are generally applicable to inverse problems with deterministic stability results. These are often available in the literature because they are also needed for convergence results of the Tikhonov regularisation (consider for example Theorem 10.4. in [17]). Finally, we complete our reasoning by proving appropriate posterior consistency results for the corresponding Bayesian nonparametric regression problem.

\section{Structure of this Article}

In Section 2, we both review preliminary material and give a detailed exposition of our main ideas, steps and results. In Section 3, we provide novel posterior consistency results for Bayesian non-parametric regression. In order to evaluate the rate for the regression problem, we compare our rates to those for Gaussian priors for which optimal rates are known. These results are needed in order to obtain posterior consistency for the elliptic inverse problem in Section 4. We obtain explicit rates for priors based on a series expansion with uniformly distributed coefficients. In Section 5, we draw a conclusion and mention other inverse problems to which this approach is applicable. The appendix contains a detailed summary of relevant technical tools such as Gaussian measures and Hilbert scales which are used in the proofs of our main results.

\section{Acknowledgments}

The author would like to thank Professor Martin Hairer, Professor Andrew Stuart, Dr. Hendrik Weber and Sergios Agapiou for helpful discussions. SJV is grateful for the support of an ERC scholarship.

\section{Preliminaries and Exposition to Posterior Consistency for Nonlinear Inverse Problems}

Our crucial idea for proving posterior consistency for a nonlinear Bayesian inverse problem is the use of stability results which allow us to break it down to posterior consistency of a Bayesian regression problem. Because the proofs are quite technical, it is worth becoming familiar with the outline of our main ideas first. Therefore this section is intended to motivate, review and summarise our investigation of posterior consistency for a nonlinear inverse problem leaving technical details to the Sections 
3 and 4. For the convenience of the reader we also repeat the relevant material on Bayesian inverse problems in Section 2.1 without proofs, thus making our exposition self-contained. In Section 2.2, we precisely define posterior consistency in this setting and place it within the literature. Subsequently, we introduce an elliptic inverse problem as guiding example for which we apply our method using stability results from [36].

Finally, we conclude our exposition by giving a general abstract theorem of posterior consistency for nonlinear inverse problems with stability results in Section 2.4.

\subsection{Summary of the Bayesian Approach to Inverse Problems on Hilbert Spaces}

The key idea of Bayesian inverse problems is to model the input $a \in X$ of a mathematical model, for example the initial condition of a PDE, as random variable with distribution $\mu_{0}(d a)$ based on a priori knowledge. This distribution is called the prior which is updated based on the observed data $y$. The resulting distribution $\mu^{y}$ is called posterior and lies in the focus of the Bayesian approach.

We assume that the data is modelled as

$$
y=\mathcal{G}(a)+\xi
$$

with $\mathcal{G}$ being the forward operator, a mapping between the Hilbert spaces $X$ and $Y$, and with the observational noise $\xi$. The aim of the inverse problem is the reconstruction of $a$ given the data $y$. Because $\mathcal{G}$ might be non-injective and $\xi$ is unknown, the problem is not exactly solvable as stated. If the distribution of the noise $\xi$ is known, then $a$ and $y$ can be treated as jointly varying random variables. Under mild assumptions on the prior, the distribution of the noise and the forward operator, there exists a conditional probability measure on $a$, called the posterior $\mu^{y}$. It is an update of the prior using the data and models the a posteriori uncertainty. Therefore it can be viewed as the solution to the inverse problem itself. In this way it is possible to obtain different explanations of the data corresponding to different modes of the posterior.

In this article, we assume that the law of the observational noise $\mu_{\xi}=\mathcal{N}(0, \Gamma)$ is a mean-zero Gaussian with covariance $\Gamma$. In this case Bayes' rule can be generalised for any $\mathcal{G}$ mapping into a finite dimensional space $Y$. It follows that

$$
\begin{aligned}
\frac{d \mu^{y}}{d \mu_{0}}(a) & \propto \exp \left(-\frac{1}{2}\|\mathcal{G}(a)-y\|_{\Gamma}^{2}\right) \propto \exp \left(-\frac{1}{2}\|\mathcal{G}(a)\|_{\Gamma}^{2}+\langle y, \mathcal{G}(a)\rangle_{\Gamma}-\|y\|_{\Gamma}^{2}\right) \\
& \propto \exp \left(-\frac{1}{2}\|\mathcal{G}(a)\|_{\Gamma}^{2}+\langle y, \mathcal{G}(a)\rangle_{\Gamma}\right) .
\end{aligned}
$$

By $\|\cdot\|_{\Gamma}$ we denote the norm of the Cameron-Martin space $\left(H_{\mu_{\xi}},\langle\cdot, \cdot\rangle_{\Gamma}\right)$ of $\mu_{\xi}$ that is the closure of $Y$ with respect to $\langle\cdot, \cdot\rangle_{\Gamma}=\left\langle\Gamma^{-1} \cdot, \cdot\right\rangle$ (see Appendix A for more details). A proper derivation of Equation (3), including the fact that its last line is also valid for functional data, and an appropriate introduction to Bayesian inverse problems can be 
found in [40] and [41]. All in all the Bayesian approach can be summarised as

$$
\begin{array}{lll}
\text { Prior } & a \sim \mu_{0} \\
\text { Noise } & \xi \sim \mathcal{N}(0, \Gamma) \\
\text { Posterior } & \frac{d \mu^{y}}{d \mu_{0}} \propto \exp \left(-\frac{1}{2}\|\mathcal{G}(a)\|_{\Gamma}^{2}+\langle y, \mathcal{G}(a)\rangle_{\Gamma}\right) .
\end{array}
$$

As one can see in this example, the posterior can usually only be expressed implicitly as an unnormalised density with respect to the prior. Thus, in order to estimate the input parameters or perform inference using the posterior, it has to be probed using either

- sampling methods, such as MCMC which aim at generating draws from the posterior or

- variational methods for determining the location of an infinitesimal ball with maximal posterior probability.

The second approach is also called the maximum a posteriori probability (MAP) estimator. It can be viewed as an extension to many classical methods for inverse problems. For example, it can be linked to the $L^{2}$-Tikhonov regularisation by considering a Gaussian prior and noise [11]. This relates the choice of norms in the Tikhonov regularisation to the choice of the covariance of the prior and the noise.

These regularisation techniques can be justified by convergence results. Similarly, inference methods based on the posterior can be justified by posterior consistency, a concept which we introduce in the next section.

\subsection{Posterior Consistency for Bayesian Inverse Problems}

As for any approach to inverse problems, the Bayesian method can be evaluated by considering an identical twin experiment. Therefore a fixed input $a^{\dagger}$, called the 'truth', is considered and data is generated using a sequence of forward models

$$
y_{n}=\mathcal{G}_{n}\left(a^{\dagger}\right)+\xi_{n}
$$

which might correspond to the increasing amount of data or diminishing noise. For each $n$ we denote the posterior corresponding to the prior $\mu_{0}$, the noise distribution $\mu_{\xi_{n}}$ and

the forward operator $\mathcal{G}_{n}$ by $\mu_{n}^{y}$. Under appropriate assumptions, the posterior $\mu_{n}^{y}$ is welldefined for $y=\mathcal{G}(a)+\xi$ given by Bayes' rule in Equation (4) for $\mu_{0}$-a.e. $a$ and $\mu_{\xi_{n}}$-a.e. $\xi_{n}$ (c.f. [41]). This Bayes' rule does not give rise to a well-defined measure for arbitrary $y$. However, we will pose assumptions such that the normalising constant in the Bayes' rule will be bounded above and below for every $a^{\dagger}$ belonging to a particular set and $\mu_{\xi_{n}}$-a.e. $y=y_{n}=\mathcal{G}_{n}\left(a^{\dagger}\right)+\xi_{n}$. We will denote these posteriors by $\mu^{y_{n}}$. This sequence of inverse problems is called posterior consistent if the posteriors $\mu^{y_{n}}$ concentrate around the 'truth' $a^{\dagger}$. We quantify the concentration by the posterior probability assigned to the ball $B_{\epsilon}^{d}$. Here $B_{\epsilon}^{d}$ denotes a ball of radius $\epsilon$ with respect to a metric $d$.

In the following we define this concept precisely and place it within the literature before closing this section by relating posterior consistency to small ball probabilities for the prior. 
Definition. (Analogue to [22]) A sequence of Bayesian inverse problems $\left(\mu_{0}, \mathcal{G}_{n}, \mathcal{L}\left(\xi_{n}\right)\right.$ ) is posterior consistent for $a^{\dagger}$ with rate $\epsilon_{n} \downarrow 0$ and with respect to a metric $d$ if for

$$
y_{n}=\mathcal{G}_{n}\left(a^{\dagger}\right)+\xi_{n},
$$

there exists a constant $M$ and a sequence $l_{n} \rightarrow 1$ such that

$$
\mathbb{P}_{\xi_{n}}\left(\mu^{y_{n}}\left(B_{M \epsilon_{n}}^{d}\left(a^{\dagger}\right)\right) \geq l_{n}\right) \rightarrow 1 .
$$

We simply say that $\left(\mu_{0}, \mathcal{G}_{n}, \mathcal{L}\left(\xi_{n}\right)\right)$ is posterior consistent if the above holds for any fixed constant $\epsilon_{n}=\epsilon>0$.

Two important special cases of this definition are

- posterior consistency in the small noise limit:

$$
\mathcal{L}\left(\xi_{n}\right)=\mathcal{L}\left(\frac{1}{\sqrt{n}} \xi\right) \text { and } \mathcal{G}_{n}=\mathcal{G}
$$

- posterior consistency in the large data limit:

$$
\mathcal{L}\left(\xi_{n}\right)=\otimes_{i=1}^{n} \mathcal{L}(\xi) \text { and } \mathcal{G}_{n}=\prod_{i=1}^{n} \mathcal{G}^{i}=\left(\mathcal{G}^{1}, \ldots, \mathcal{G}^{n}\right) .
$$

In the above formulation $\mathcal{G}^{i}$ corresponds to different measurements while $\mathcal{L}(\cdot)$ denotes the law of a random variable.

There exists a variety of results for posterior consistency and inconsistency for statistical problems. Two important examples are the identification of a distribution from (often i.i.d.) samples or density estimation [14, 22, 43, 28]. The former is concerned with considering a prior on a set of probability distributions and the resulting posterior based on $n$ samples of one of these probability distributions. In [16], Doob proved that if a countable collection of samples almost surely allows the identification of the generating distribution, then the posterior is consistent for almost every probability distribution with respect to the prior. This very general result is not completely satisfactory because it does not provide a rate and the interest may lie in showing posterior consistency for every possible truth in a certain class. Moreover, some surprisingly simple examples of posterior inconsistency have been provided for example by considering distributions on $\mathbb{N}$ [21]. The necessary bounds for posterior consistency (c.f. Equation (5)) can be obtained using the existence of appropriate statistical tests which are due to bounds on entropy numbers. These methods are used in a series of articles, for example in [22, 39, 43, 23]. This idea has also recently been applied to the Bayesian approach to linear inverse problems in 35].

In general, posterior consistency for infinite dimensional inverse problems has mostly been studied for linear inverse problems in the small noise limit where the prior is either a sieve prior, a Gaussian or a wavelet expansion with uniform distributed coefficients [30, 1, 35, 20]. Except for [35], all these articles exploit the explicit structure of the posterior in the conjugate Gaussian setting, that means that we have a Gaussian prior as well as a Gaussian posterior.

In contrast, we consider general priors, general forward operators and Gaussian noise in this article. Usually, the posterior has a density with respect to the prior as 
in Equation (4). However, it is possible to provide examples where both the prior and posterior are Gaussian but not absolutely continuous. This can be achieved using for example Proposition 3.3 in [2].

Subsequently, we assume that the posterior has a density with respect to the prior implying that the posterior probability of a set is zero whenever the prior probability of this set is zero. Therefore it is necessary that $a^{\dagger}$ is in the support of the prior giving rise to the following definition.

Definition. The support of a measure $\mu$ in a metric space $(X, d)$ is given by

$$
\operatorname{supp}_{d}(\mu)=\left\{x \mid \mu\left(B_{\epsilon}^{d}(x)>0 \forall \epsilon>0\right)\right\} .
$$

It is natural to expect that the posterior consistency rate depends on the behaviour of $\mu_{0}\left(B_{\epsilon}^{d}\left(a^{\dagger}\right)\right)$ as $\epsilon \rightarrow 0$. Asymptotics of this type are called small ball probabilities. We recommend [32] as a good survey and refer the reader to [34] for an up-to-date list of references. In this article, we consider algebraic rates of posterior consistency, that means we take $\epsilon_{n}=n^{-\kappa}$ in Definition 2.2. In order to establish these rates of posterior consistency, we consider small ball asymptotics of the following form

$$
\log \left(\mu_{0}\left(B_{\epsilon}^{d}\left(a^{\dagger}\right)\right) \succsim-\epsilon^{-\rho}\right.
$$

where $\rho>0$ and with the notation as in Appendix Appendix A.

Both posterior consistency and the contraction rate depend on properties of the prior. This suggests that we should choose a prior with favourable posterior consistency properties. From a dogmatic point of view the prior is only supposed to be chosen to match the subjective a priori knowledge. In practice priors are often picked based on their computational performance whereas some of their parameters are adapted to represent the subjective knowledge. An example for this is the choice of the base measure and the intensity for a Dirichlet process [28].

Finally, we would like to conclude this Section by mentioning that it has been shown in [15] that posterior consistency is equivalent to the property that the posteriors corresponding to two different priors merge. The yet unpublished book [23] contains a more detailed discussion about the justification of posterior consistency studies for dogmatic Bayesians.

\subsection{An Elliptic Inverse Problem as an Application of our Theory}

The aim of this section is to set up the elliptic inverse problem for which we will prove posterior consistency (c.f. Section 2.2) both in the small noise and the large data limit. In a second step we describe the available stability results and how they can be used to reduce the problem of posterior consistency of a nonlinear inverse problem to that of a linear regression problem. We end this section by stating a special case of our posterior consistency results in Section 4.

Our results do not only apply to this particular elliptic inverse problem but to any nonlinear inverse problem with appropriate stability results (c.f. Section 2.4). However, 
the results for the elliptic inverse problem are of particular interest because it is used in oil reservoir simulations and the reconstruction of the groundwater flow [46, 36, 27].

The forward model corresponding to our elliptic inverse problem is based on the relation between $p$ and $a$ given by the elliptic PDE in 1 .

We would like to highlight that the relation between $a$ and $p$ is nonlinear. Under the following assumptions, the solution operator $p(x ; a)$ to the above PDE is well-defined [24].

Assumption 1. (Forward conditions) Suppose that

(i) D is compact, satisfies the exterior sphere condition (see [24]) and has a smooth boundary;

(ii) $a \in C^{1}(D) \cap C(\bar{D})$ and $f$ is smooth in $\bar{D}$;

(iii) $a>a_{\text {min }}>0$ and $f>f_{\text {min }}>0$ in Equation (1).

Under these assumptions, the regularity results from [24] yield the following forward stability result.

Proposition 2.1. If $a_{1}$ and $a_{2}$ satisfy Assumption 1 and are elements of $C^{\alpha}$ for $\alpha \geq 1$, then

$$
\left\|p\left(\cdot ; a_{1}\right)-p\left(\cdot ; a_{2}\right)\right\|_{C^{\alpha+1}} \leq M\left\|a_{1}-a_{2}\right\|_{C^{\alpha}} .
$$

The inverse problem is concerned with the reconstruction of $a$ given the data

$$
y_{n}=\mathcal{G}_{n}(a)+\xi,
$$

which is related to $p$ in the following way.

Assumption 2. The forward operator $\mathcal{G}$ can be split into a composition of the solution operator $p$ and an observation operator $\mathcal{O}$, that is

$$
\mathcal{G}_{n}(a)=\mathcal{O}_{n}(p(\cdot ; a)) .
$$

The Bayesian approach to the Elliptic Inverse Problem (EIP) summarises as

\begin{tabular}{|cc|}
\hline Model & $-\nabla \cdot(a \nabla p(\cdot ; a))=f(x)$ in $D, \quad p=0$ on $\partial D$ \\
Prior & $\mu_{0}$ on $a$ \\
Data & $y=\mathcal{G}_{n}(a)+\xi_{n}=\mathcal{O}_{n}(p(\cdot, a))+\xi_{n}, \xi_{n} \sim \mathcal{N}\left(0, \Gamma_{n}\right)$ \\
Posterior & $\frac{d \mu^{n}}{d \mu_{0}}(a) \propto \exp \left(-\frac{1}{2}\left\|\mathcal{G}_{n}(a)\right\|_{\Gamma_{n}}^{2}+\langle y, \mathcal{G}(a)\rangle_{\Gamma_{n}}\right)$.
\end{tabular}

A rigorous Bayesian formulation of this inverse problem, with log-Gaussian priors and Besov priors has been given in [12] and [10] respectively. In [38] the problem is considered with a prior based on a series expansion with uniformly distributed coefficients (see Section 4.1.1). In the same article, a generalised Polynomial Chaos (gPC) method is derived in order to approximate posterior expectations.

We consider posterior consistency as set up in Definition 2.2 in the following cases: 
- the small noise limit with $\mathcal{O}_{n}=$ Id corresponding to a functional observation and an additive Gaussian random field as noise such that

$$
y_{n}=p(\cdot ; u)+\frac{1}{\sqrt{n}} \xi
$$

- the large data limit with $\mathcal{O}_{n}=\left(e_{x_{i}}\right)_{i=1}^{n}$ where $e_{x_{i}}$ are evaluations at $x_{i} \in D$. In this case the data takes the form

$$
y_{n}=\left\{p\left(x_{i} ; a\right)\right\}_{i=1}^{n}+\xi_{n} .
$$

Posterior consistency in both cases are based on a stability result which can be derived by taking $a$ as the unknown in Equation (1). This leads to the following hyperbolic PDE

$$
-\nabla a \cdot \nabla p-a \Delta p=f .
$$

Imposing Assumption 1, it has been established that there exists a unique solution $a$ to this PDE without any additional boundary conditions:

Proposition 1 (Corollary 2 on page 220 in [36]). Suppose $p$ arises as a solution to Equation (1) with a as diffusion coefficient satisfying Assumption 1. Then Equation (8) is uniquely solvable for any $f \in L^{\infty}(D)$ and a such that

$$
\|a\|_{\infty} \leq D\left(a_{\min }, f_{\min },\|\nabla a\|_{\infty}\right)\|p\|_{\infty} .
$$

Moreover, if $a_{1}$ and $a_{2}$ satisfy these assumptions, then

$$
\left\|a_{1}-a_{2}\right\|_{\infty} \leq M\left\|a_{1}\right\|_{C^{1}} \cdot\left\|p\left(\cdot, a_{1}\right)-p\left(\cdot, a_{2}\right)\right\|_{C^{2}} .
$$

The stability result above and a change of variables (Theorem Appendix B.1 implies

$$
\mu^{y_{n}}\left(B_{\epsilon}^{L^{\infty}}\left(a^{\dagger}\right)\right)=\tilde{\mu}^{y_{n}}\left(p\left(B_{\epsilon}^{L^{\infty}}\left(a^{\dagger}\right)\right) \geq \tilde{\mu}^{y_{n}}\left(B_{\frac{\epsilon}{M}}^{C^{2}}\left(p^{\dagger}\right)\right) .\right.
$$

This statement reduces posterior consistency of the EIP in $L^{\infty}$ to posterior consistency of the following Bayesian Regression Problem (BRP) in $C^{2}$

$$
\begin{array}{ccc}
\text { Prior } & \tilde{\mu}_{0}=p_{\star} \mu_{0} \text { on } p \\
\text { Data } & y=\mathcal{O}_{n}(p)+\xi_{n}, \xi_{n} \sim \mathcal{N}\left(0, \Gamma_{n}\right) \\
\text { Posterior } & \frac{d \tilde{\mu}^{y_{n}}}{d \tilde{\mu}_{0}}(p) \propto \exp \left(-\frac{1}{2}\|\mathcal{O}(p)\|_{\Gamma_{n}}^{2}+\langle y, \mathcal{O}(p)\rangle_{\Gamma_{n}}\right) \\
& & \text { with } \mathcal{O}_{n}=\operatorname{Id} \text { or } \mathcal{O}_{n}=\left(e_{x_{i}}\right)_{i=1}^{n}
\end{array}
$$

where $p$ is now treated as an variable, that is the prior and the posterior are now formulated on the pressure space. Moreover, $p_{\star} \mu_{0}$ denotes the push forward of the prior under $p$. Note that for $\mathcal{O}_{n}=\mathrm{Id}$ the BRP can also be viewed as the simplest linear inverse problem.

The required posterior consistency results for the BRP can be derived from those in Section 3 using interpolation inequalities. In this way we obtain posterior consistency results in Section 4 a special case of which is the following theorem: 
Theorem 2.2 (4.1). Suppose that the prior $\mu_{0}$ satisfies

$$
a(x) \geq \lambda>0 \quad \forall x \in D \text { and }\|a\|_{C^{\alpha}} \leq \Lambda \quad \text { for } \mu_{0} \text {-a.e. a and for } \alpha>1
$$

Let the noise be given by $\xi \sim \mathcal{N}\left(0,\left(-\Delta_{\text {Dirichlet }}\right)^{-r}\right)$. If $\alpha>r+\frac{d}{2}-2$ and $\alpha>r-1$, then (EIP) is posterior consistent for any $a^{\dagger} \in$ supp $_{C^{\alpha}} \mu_{0}$ in the small noise limit with respect to the $C^{\tilde{\alpha}}$-norm for any $\tilde{\alpha}<\alpha$.

This approach is not limited to the EIP as the following section shows.

\subsection{Posterior Consistency through Stability Results}

In Section 2.3, we present our main idea, that is the reduction of the problem of posterior consistency of the EIP to that of the BRP. The main ingredients of this reduction are the stability result that was summarised in Proposition 1 and the posterior consistency results for the BRP. This approach is not limited to the EIP but it is applicable to any inverse problem for which appropriate stability results are available. This is the case for many inverse problems such as the inverse scattering problem in [31] or the Calderon problem in [3]. We would like to point out that these stability results are also crucial for proving the convergence of regularisation methods (see Theorem 10.4 in [17]).

Theorem 2.3. Suppose $\mathcal{G}_{n}=\mathcal{O}_{n} \circ G$ with $G:\left(X,\|\cdot\|_{X}\right) \rightarrow\left(Y,\|\cdot\|_{Y}\right)$ and $\mathcal{O}_{n}:\left(Y,\|\cdot\|_{Y}\right) \rightarrow\left(Z,\|\cdot\|_{Z}\right)$. Moreover, we assume that

- there exists a stability result of the form

$$
\begin{aligned}
& \left\|a_{1}-a_{2}\right\|_{X} \leq b\left(\left\|G\left(a_{1}\right)-G\left(a_{2}\right)\right\|_{Y}\right) \\
& \text { where } b: \mathbb{R}^{+} \rightarrow \mathbb{R}^{+} \text {is increasing and, } b(0)=0 ;
\end{aligned}
$$

- the sequence of Bayesian inverse problems $\left(G_{\star} \mu_{0}, \mathcal{O}_{n}, \mathcal{L}\left(\xi_{n}\right)\right)$ is posterior consistent with respect to $\|\cdot\|_{Y}$ for all $p^{\dagger} \in A$ with rate $\epsilon_{n}$.

Then $\left(\mu_{0}, \mathcal{G}_{n}, \mathcal{L}\left(\xi_{n}\right)\right)$ is posterior consistent with respect to $\|\cdot\|_{X}$ for all $a^{\dagger} \in G^{-1}(A)$ with rate $b\left(\epsilon_{n}\right)$.

Proof. Using the notation of Section 2.3, we denote the posteriors for the Bayesian inverse problems $\left(\mu_{0}, \mathcal{G}_{n}, \mathcal{L}\left(\xi_{n}\right)\right)$ and $\left(G_{\star} \mu_{0}, \mathcal{O}_{n}, \mathcal{L}\left(\xi_{n}\right)\right)$ by $\mu^{y_{n}}$ and $\tilde{\mu}^{y}$, respectively. Then a change of variables (c.f. Theorem Appendix B.1) implies

$$
\mu^{y}\left(B_{b\left(\epsilon_{n}\right)}^{X}\left(a^{\dagger}\right)\right) \geq \tilde{\mu}^{y}\left(B_{\epsilon_{n}}^{Y}\left(G\left(a^{\dagger}\right)\right)\right)
$$

\section{Posterior Consistency for Bayesian Regression}

As described in the previous section, for many inverse problems posterior consistency can be reduced to posterior consistency of a BRP (c.f. Section 2.4) using stability results. Thus, with the results obtained in this section we may conclude posterior consistency for apparently harder nonlinear inverse problems. For the EIP this is achieved by an 
application of the results in Theorem 3.3 and 3.7. Because the derivation of these two results is quite technical, we first give a summary and we recommend the reader to become familiar with both theorems but to skip the technical details on the first read.

It is classical to model the response as

$$
y_{n}=\mathcal{O}_{n}(p)+\xi_{n} .
$$

In the following we consider two Bayesian regression models with

- $\mathcal{O}_{n}=$ Id and the noise is a Gaussian random field that is scaled to zero like $\xi_{n}=n^{-\frac{1}{2}} \xi$ or

- $\mathcal{O}_{n}=\left(e_{x_{i}}\right)_{i=1}^{n}$ and $\mathcal{L}\left(\xi_{n}\right)=\otimes_{i=1}^{n} \mathcal{N}\left(0, \sigma^{2}\right)$ corresponding to evaluations of a function with additive i.i.d. Gaussian noise.

These models represent the large data and the small noise limit, respectively.

We prove posterior consistency for both problems under weak assumptions on the prior. This is necessary because the BRPs resulting from nonlinear inverse problems are usually only given in an implicit form. For both cases we are able to obtain a rate assuming appropriate asymptotic lower bounds on the small ball probabilities of the prior around $a^{\dagger}$ (see Section 2.2). Moreover, posterior consistency with respect to stronger norms can be obtained using prior or posterior regularity in combination with interpolation inequalities which is the subject of Section 3.3 .

For the large data limit, that is $\mathcal{O}_{n}=\left(e_{x_{i}}\right)_{i=1}^{n}$, we obtain posterior consistency with respect to the $L^{\infty}$-norm in Section 3.2. We assume an almost sure upper bound on a Hlder norm for the prior and an additional condition on the locations of the observations. The latter is justified by construction of a counterexample.

For the small noise limit, that is $\mathcal{O}_{n}=\mathrm{Id}$, we prove posterior consistency with respect to the Cameron-Martin norm of the noise in Section 3.1. This norm corresponds to the $\|\cdot\|_{1}$-norm in the Hilbert scale with respect to the covariance operator $\Gamma$. Both the Cameron-Martin norm and Hilbert scales are introduced in Appendix A. If an appropriate $\|\cdot\|_{s}$-norm is $\mu_{0}$-a.s. bounded, we obtain an explicit rate of posterior consistency. Otherwise, the rate is implicitly given as a low-dimensional optimisation problem. However, the condition for mere posterior consistency takes a simple form.

Corollary 3.1. (See Corollary 3.5 for the case of general noise) Suppose that the noise is given by $\xi \sim \mathcal{N}\left(0,(-\Delta)^{-r}\right)$ and $\mu_{0}\left(\exp \left(f\|p\|_{H^{s}}^{e}\right)\right)<\infty$ for $s>r+\frac{d}{2}$ and $f>0$. Then the posterior is consistent in $H^{r}$ for any $a^{\dagger} \in$ supp $_{\mathcal{H}^{r}}$ if $e$ and $\lambda=\frac{s-r-\frac{d}{2}}{s-r}$ satisfy the following conditions

$$
\begin{array}{cc}
e>-1+\sqrt{8-8 \lambda} & \text { if } \lambda \in\left[0, \frac{1}{2}\right] \\
e>2-2 \lambda & \text { if } \lambda \in \frac{1}{2}, 1 .
\end{array}
$$

Remark 1. If the prior is Gaussian, then the above inequality is satisfied because $e=2$ and the RHS is less than 2 for any $\lambda \in(0,1)$. Thus, the only remaining condition is $s>r+\frac{d}{2}$. 
Remark 2. It is worth pointing out that for the large class of log-concave measures it is known that $e \geq 1$, for details consult [5].

In the statistics literature regression models are mainly concerned with pointwise observations. Despite its name this is also true for functional data analysis (see [19]). However, the regression problem associated with $\mathcal{O}_{n}=\mathrm{Id}$ can be viewed as a particular linear inverse problem. As described in the introduction, this has been studied for Gaussian priors in [30] and [1]. Although our focus lies on establishing posterior consistency for general priors and non-linear models, we also obtain rates which in the special case of Gaussian priors are close to the optimal rates given in the references above.

\subsection{The Small Noise Limit for Functional Response}

In the following we study posterior consistency for a Bayesian regression problem assuming that the data takes values in the Hilbert space $H$. In particular we deal with the regression model

$$
y=a+\frac{1}{\sqrt{n}} \xi
$$

with $y, a$ and $\xi$ all being elements of $H$. Moreover, we suppose that the observational noise $\xi$ is a Gaussian random field $\mu_{\xi}=\mathcal{N}(0, \Gamma)$ on $H$ and we assume that it satisfies the following assumption.

Assumption 3. Suppose there is $\sigma_{0} \geq$ such that $\Gamma^{\sigma}$ is trace-class for all $\sigma>\sigma_{0}$, that is

$$
\sum_{k=1}^{\infty} \lambda_{k}^{2 \sigma}<\infty
$$

Imposing this assumption, it becomes possible to quantify the regularity of the observational noise in terms of the Hilbert scale defined with respect to the covariance operator (c.f. Appendix A. More precisely, this is possible due to Lemma Appendix A.2. from [1].

The regression model in Equation (9) is a special case of a general inverse problem as considered in Equation (2). Hence the corresponding posterior takes the following form (c.f. Equation (4)).

$$
\frac{d \mu^{y}}{d \mu_{0}}=Z(n, \xi) \exp \left(-\frac{1}{2} n\|a\|_{1}^{2}+n\langle a, y\rangle_{1}\right) \text {. }
$$

Assuming that the data takes values in the Hilbert space $H$, Equation (10) can simply be derived by an application of the Cameron-Martin lemma in combination with the conditioning lemma (Lemma 5.3 in [26]). We generate data for a fixed 'truth' $a^{\dagger}$

$$
y=a^{\dagger}+\frac{1}{\sqrt{n}} \xi .
$$


By changing the normalising constant, we may rewrite the posterior in the following way

$$
\frac{d \mu^{y}}{d \mu_{0}}=Z(n, \xi) \exp \left(-\frac{n}{2}\left\|a-a^{\dagger}\right\|_{1}^{2}+\sqrt{n}\left\langle a-a^{\dagger}, \xi\right\rangle_{1}\right) .
$$

The normalising constant is bounded above and below for $y_{n}=\mathcal{G}_{n}\left(a^{\dagger}\right)+\xi_{n}$ for $\mu_{\xi_{n}}$-a.e. $\xi$. In fact, this holds under weaker assumptions than needed for our results.

Lemma 3.2. Suppose $\mu_{0}\left(\exp \left(f\|a\|_{s}^{e}\right)\right)<\infty$ for $s>1+\sigma_{0}$ and $e>\frac{2 \sigma_{0}}{s-1+\sigma_{0}}$. Then the normalising constant in Equation (12) is bounded for $\mu_{\xi_{n}}$-a.s. and every $a^{\dagger} \in \mathcal{H}^{s}$ above and away form zero.

Proof. See Appendix D.

The expression above suggests that the posterior concentrates in balls around the truth in the Cameron-Martin norm. First, we make this fact rigorous for priors which are a.s. uniformly bounded with respect to the $\|\cdot\|_{s}$-norm. In a second step, we assume that the prior has higher exponential moments. Considering Gaussian priors, we show that our rate is close to the optimal rate obtained in [30].

3.1.1. Posterior Consistency for Uniformly Bounded Priors The following theorem can be viewed as a preliminary step towards Theorem 3.4 which contains our most general posterior consistency result for the Bayesian regression problem in the small noise limit. While containing our main ideas, the following result also establishes an explicit rate for posterior consistency which will be used for the EIP in Section 4.

Theorem 3.3. Suppose that the noise satisfies Assumption 3 and

$$
\|a\|_{s} \leq U \mu_{0} \text {-a.s. }
$$

for $s>1+\sigma_{0}$. If $a^{\dagger} \in \operatorname{supp}_{\mathcal{H}^{1}}\left(\mu_{0}\right)$ and $a^{\dagger} \in \mathcal{H}^{s}$, then $\mu^{y_{n}}$ is consistent in $\mathcal{H}^{1}$. Additionally, if the following small ball asymptotic is satisfied

$$
\log \left(\mu_{0}\left(B_{\epsilon}^{1}\left(a^{\dagger}\right)\right) \succsim-\epsilon^{-\rho}\right.
$$

then this holds with rate $M n^{-\kappa}$ for any $\kappa<\min \left\{\frac{1}{2(2-\lambda)}, \frac{1}{2+\rho}\right\}$ with $\lambda=\frac{s-1-\sigma_{0}}{s-1}$.

Proof. Our proof is based on the observation that posterior consistency is implied by the existence of a sequence of subsets $S_{n}$ such that $\mu_{\xi}\left(S_{n}\right) \rightarrow 1$ and

$$
\sup _{\xi \in S_{n}} \frac{\mu^{y_{n}}\left(B_{\epsilon n^{-\kappa}}^{1}\left(a^{\dagger}\right)^{c}\right)}{\mu^{y_{n}}\left(B_{\epsilon n^{-\kappa}}^{1}\left(a^{\dagger}\right)\right)} \rightarrow 0 \text { for } n \rightarrow \infty
$$

where $y_{n}=a^{\dagger}+\frac{1}{\sqrt{n}} \xi$. This implication holds because

$$
\mu^{y_{n}}\left(B_{\epsilon n^{-\kappa}}^{1}\left(a^{\dagger}\right)\right)+\mu^{y_{n}}\left(B_{\epsilon n^{-\kappa}}^{1}\left(a^{\dagger}\right)^{c}\right)=1
$$

and thus

$$
\sup _{\xi \in S_{n}} \frac{\mu^{y_{n}}\left(B_{\epsilon n^{-\kappa}}^{1}\left(a^{\dagger}\right)^{c}\right)}{\mu^{y_{n}}\left(B_{\epsilon n^{-\kappa}}^{1}\left(a^{\dagger}\right)\right)} \leq \delta \Rightarrow \frac{1}{1+\delta} \leq \sup _{\xi \in S_{n}} \mu^{y_{n}}\left(B_{\epsilon n^{-\kappa}}^{1}\left(a^{\dagger}\right)\right)
$$


which together with $\mu_{\xi}\left(S_{n}\right) \rightarrow 1$ implies posterior consistency, for details see Equation (5).

Fix $\gamma>0$. Then $S_{n}=B_{K_{n}^{\prime}}^{1-\sigma_{0}-\gamma}(0)$ with $K_{n}^{\prime} \uparrow \infty$ as $n \rightarrow \infty$ sufficiently slow. We notice that Lemma Appendix A.2 implies that $\mathbb{P}_{\xi}\left(\xi \in B_{K_{n}^{\prime}}^{1-\sigma_{0}-\gamma}(0)\right) \rightarrow 1$ as $n \rightarrow \infty$. The remainder of the proof will be devoted to showing that Equation 15 holds. We bound $\left\langle a-a^{\dagger}, \xi\right\rangle_{1}$ by smoothing $\xi$ at the expense of $a-a^{\dagger}$

$$
\begin{aligned}
\left|\left\langle a-a^{\dagger}, \xi\right\rangle_{1}\right| & \leq\left|\left\langle\Gamma^{-1+\frac{1-\sigma_{0}-\gamma}{2}}\left(a-a^{\dagger}\right), \Gamma^{\frac{\sigma_{0}-1+\gamma}{2}} \xi\right\rangle_{1}\right| \\
& \leq\left\|a-a^{\dagger}\right\|_{1+\sigma_{0}+\gamma}\|\xi\|_{1-\sigma_{0}-\gamma} \\
& \leq\left\|a-a^{\dagger}\right\|_{1+\sigma_{0}+\gamma} K_{n}^{\prime} \forall \xi \in B_{K_{n}^{\prime}}^{1-\sigma_{0}}(0) .
\end{aligned}
$$

Interpolating between $\mathcal{H}^{1}$ and $\mathcal{H}^{s}$ for $s$ (c.f. Lemma Appendix A.1) yields

$$
\left|\left\langle a-a^{\dagger}, \xi\right\rangle_{1}\right| \leq K_{n}^{\prime}\left\|a-a^{\dagger}\right\|_{1}^{\lambda}\left\|a-a^{\dagger}\right\|_{s}^{1-\lambda} \leq K_{n}\left\|a-a^{\dagger}\right\|_{1}^{\lambda}
$$

with $\lambda=\frac{s-1-\sigma_{0}-\gamma}{s-1}$. An application of Equation $\sqrt{12}$ yields the following upper bound

$$
\begin{aligned}
\mu^{y}\left(B_{\frac{\epsilon}{n^{\kappa}}}^{1}\left(a^{\dagger}\right)\right) & \geq Z(n, \xi) \inf _{a \in B_{\frac{\epsilon}{2} n^{-\kappa}}^{1}} \exp \left(-n\left\|a-a^{\dagger}\right\|_{1}^{2}-\sqrt{n}\left\langle a-a^{\dagger}, \xi\right\rangle_{1}\right) \mu_{0}\left[B_{\frac{\epsilon}{2} n^{-\kappa}}^{1}\left(a^{\dagger}\right)\right] \\
& \geq Z(n, \xi) \exp \left[-n^{1-2 \kappa}\left[\frac{\epsilon\left\|a-a^{\dagger}\right\|_{1}}{2}\right]^{2}-K_{n} n^{\frac{1}{2}-\lambda \kappa}\left(\frac{\epsilon}{2}\right)^{\lambda}\right] \mu_{0}\left[B_{\frac{\epsilon}{2} n^{-\kappa}}^{1}\left(a^{\dagger}\right)\right] .
\end{aligned}
$$

Similarly, we obtain the following upper bound

$$
\mu^{y}\left(B_{\epsilon n^{-\kappa}}^{1}\left(a^{\dagger}\right)\right) \leq Z(n, \xi) \sup _{a \in B_{\epsilon n^{-\kappa}}^{1}\left(a^{\dagger}\right)} \exp \left(-n\left\|a-a^{\dagger}\right\|_{1}^{2}+K_{n} \sqrt{n}\left\|a-a^{\dagger}\right\|_{1}^{\lambda}\right)
$$

The expression in the exponential in Equation 12 can be rewritten as a function $f(d)=-n d^{2}+K_{n} n^{\frac{1}{2}} d^{\lambda}$ of $d=\left\|a-a^{\dagger}\right\|$ which is decreasing on $\left[\left(K_{n} \lambda n^{-\frac{1}{2}} / 2\right)^{\frac{1}{2-\lambda}}, \infty\right)$. If

$$
-\frac{1}{2(2-\lambda)}<-\kappa
$$

then $\left\|a-a^{\dagger}\right\|_{1}^{2} \in\left[\left(K_{n} \lambda n^{-\frac{1}{2}} / 2\right)^{\frac{1}{2-\lambda}}, \infty\right)$ for $a \in B_{\epsilon n^{-\kappa}}^{1}\left(a^{\dagger}\right)$ and $n$ large enough leading to

$$
\mu^{y}\left(B_{\epsilon n^{-\kappa}}^{1}\left(a^{\dagger}\right)\right) \leq Z(n, \xi) \exp \left(-\epsilon^{2} n^{1-2 \kappa}+n^{\frac{1}{2}-\kappa \lambda} \epsilon^{\lambda} K_{n}\right) .
$$

We now derive sufficient conditions for $n^{1-2 \kappa}$ to be the dominant term in the exponential in the Equations (18) and (20) implying Equation (15). This is the case if, in addition to Inequality (19),

$$
\begin{array}{ll}
1-2 \kappa & >\frac{1}{2}-\kappa \lambda \text { and } \\
\log \mu_{0}\left(B_{\frac{\epsilon n-\kappa}{2}}^{1}\left(a^{\dagger}\right)\right) & \gtrsim-n^{1-2 \kappa}
\end{array}
$$


hold. The first line is equivalent to Inequality (19) and using Inequality (14) the second line is implied by

$$
1-2 \kappa>\kappa \rho .
$$

Thus, the Inequalities $(19)$ and $(21)$ imply that $-n^{1-2 \kappa}$ is the dominant term in the Inequalities (18) and (20) establishing Equation (15). Letting $\gamma \rightarrow 0$ concludes the proof.

3.1.2. Extension to the Case of Unbounded Priors In the following we weaken the assumptions of Theorem 3.3 by assuming that the prior has exponential moments of $\|\cdot\|_{s}^{e}$. The price we pay is that the algebraic rate of convergence is implicitly given as a low-dimensional optimisation problem.

Theorem 3.4. Suppose that the noise satisfies Assumption 3, the prior satisfies the small ball asymptotic

$$
\log \left(\mu_{0}\left(B_{\epsilon}^{1}\left(a^{\dagger}\right)\right) \succsim-\epsilon^{-\rho}\right.
$$

and $\int \exp \left(3 f\|a\|_{s}^{e}\right) d \mu_{0}(a)<\infty$ for $f>0$ and $e>0$ for $s>1+\sigma_{0}$. If the following optimisation problem has a solution $\kappa^{\star}>0$, then for any $\kappa<\kappa^{\star}$ the posterior $\mu^{y_{n}}$ is consistent in $\mathcal{H}^{1}$ for $a^{\dagger}$ in $\mathcal{H}^{s}$ with rate $n^{-\kappa}$.

Maximize $\kappa$ with respect to $\kappa, p \geq 1, \eta, \theta \geq 0$ subject to

$$
\begin{aligned}
\frac{1}{2}+\eta \frac{p}{q}-\kappa \lambda p & <1-2 \kappa \\
\frac{1}{2}-\eta+(1-\lambda) q \theta & <1-2 \kappa \\
\rho \kappa & <e \theta \\
\rho \kappa & <1-2 \kappa \\
\lambda p & <2 \\
\left(\eta \frac{p}{q}-\frac{1}{2}\right) \frac{1}{2-\lambda p} & <-\kappa \\
(1-\lambda) q & <e
\end{aligned}
$$

where $\lambda:=\frac{s-1+\sigma_{0}}{s-1}$.

Proof. See Appendix Appendix C.

Remark 3. In general, e(s) might depend on $s$ for $\int \exp \left(3 f\|a\|_{s}^{e}\right) d \mu_{0}(a)<\infty$ to hold. Therefore the rate might be improved by optimising over different $s>1+\sigma_{0}$.

Whereas the algebraic rate in Theorem 3.4 is implicit, the following corollary yields a simple condition implying posterior consistency. 
Corollary 3.5. Suppose that the noise satisfies Assumption 3, $a^{\dagger} \in \operatorname{supp}_{\mathcal{H}^{1}}\left(\mu_{0}\right)$ and $\int \exp \left(3 f\|a\|_{s}^{e}\right) d \mu_{0}(a)<\infty$ for $f>0, e>0$ and $s>1+\sigma_{0}$. If one of the following two conditions holds

$$
\begin{array}{ll}
0<\lambda \leq \frac{1}{2} \text { and } & e>-1+2 \sqrt{2} \sqrt{1-\lambda} \quad \text { or } \\
\frac{1}{2}<\lambda<1 \text { and } & e>2-2 \lambda,
\end{array}
$$

then $\mu^{y_{n}}$ is posterior consistent for $a^{\dagger}$ in $\mathcal{H}^{s}$.

Proof. It follows from the proof of Theorem 3.4 that we only have to find $\eta, \theta \geq 0, p \geq 1$ and $s$ such that the Inequalities (C.3), (C.4), (C.8), (C.13) and (C.16) are satisfied. Choosing $\eta$ as large as Inequality C.3 permits, that is $\eta:=\frac{1}{2(p-1)}-\epsilon$, extends the range of solutions of the other inequalities $(\sqrt{\mathrm{C} .4})$ and $(\mathrm{C} .16)$ ) containing $\eta$. Similarly, choosing $\theta$ as large as C.4 permits, that is $\theta:=\frac{0.5+\eta}{(1-\lambda) q}-\epsilon$, extends the range of solutions of Inequality (C.16). Letting $\epsilon \rightarrow 0$ in (C.16) yields

$$
\begin{aligned}
& p \quad \geq 1 \\
& \lambda p \quad<2 \\
& (1-\lambda) q<e \\
& \frac{(p-2)\left(\frac{p-1}{e(p-1)+(\lambda-1) p}+1\right)}{2(p-1)}<\max \left(1, \frac{e}{2-2 \lambda}\right) .
\end{aligned}
$$

Now it is left to perform a case-by-case analysis. Starting from Inequality (22), the first two cases are $\frac{e}{2-\lambda}<1$ and $\frac{e}{2-\lambda} \geq 1$. For these cases we have to treat $e(-1+p)+p(-1+\lambda)<0$ and $e(-1+p)+p(-1+\lambda) \geq 0$ separately in order to rearrange Equation (22) to a quadratic inequality in $p$. The details are tedious but straightforward algebra.

Remark 4. We would like to point out that the Remarks 1 and 2 are also valid for this more general Corollary 3.5 .

3.1.3. Comparison for the Special Case of Gaussian Priors In the special case of jointly diagonalisable prior and noise covariance, we evaluate the consistency rate in Theorem 3.4 by comparing it with the optimal rates obtained in [30]. By numerically solving the optimisation problem in Theorem 3.4, we indicate that our rates are close to the optimal rate.

In the following we first derive a Gaussian prior and noise for a regression problem before reformulating our result in this context. In a second step we reformulate the problem in the notation of [30] and state the corresponding result. We conclude this section by an actual comparison between the posterior consistency rate obtained in [30] and the results of this paper.

We suppose that the prior is Gaussian $\mu_{0}=\mathcal{N}\left(0, \mathcal{C}_{0}\right)$ and that the covariance operators $\mathcal{C}_{0}$ of the prior and $\Gamma$ of the noise are jointly diagonalisable over $\left\{e_{i}\right\}$ denoting 
an orthonormal basis of eigenvectors. Furthermore, we assume that the eigenvalues $\mu_{j}^{2}$ and $\lambda_{j}^{2}$ of $\mathcal{C}_{0}$ and $\Gamma$ satisfy

$$
\begin{aligned}
& \mu_{j}=j^{-t} \\
& \lambda_{j}=j^{-r},
\end{aligned}
$$

respectively. The inner product of the Hilbert scale with respect to $\Gamma$ can now explicitly be written as

$$
\langle x, y\rangle_{r}=\sum_{j=1}^{\infty} \mu_{j}^{-2 r} x_{j} y_{j}, \quad\|x\|_{r}^{2}=\sum_{j=1}^{\infty} \mu_{j}^{-2 r} x_{j}^{2} .
$$

Moreover, we remark that Assumption 3 is satisfied with $\sigma_{0}=\frac{1}{2 r}$. The covariance operator $\tilde{\mathcal{C}_{0}}$ of $\mu_{0}$ on $\mathcal{H}^{s}$ has eigenvalues $\left.\mu_{j}\right|_{\mathcal{H}^{s}}=j^{-t+r s}$ which can be seen by denoting $S^{a} e_{k}:=k^{a} e_{k}$ and calculating

$$
\begin{aligned}
\mathbb{E}_{\mu_{0}}\langle x, u\rangle_{\mathcal{H}^{s}}\langle x, v\rangle_{\mathcal{H}^{s}} & =\mathbb{E}_{\mu_{0}}\left\langle x, S^{2 s r} u\right\rangle_{\mathcal{H}}\left\langle x, S^{2 s r} v\right\rangle_{\mathcal{H}} \\
& =\left\langle\mathcal{C}_{0} S^{2 s r} u, S^{2 s r} u\right\rangle_{\mathcal{H}}=\left\langle S^{2 s r} \mathcal{C}_{0} u, v\right\rangle_{\mathcal{H}^{s}} .
\end{aligned}
$$

In order to conclude that $\tilde{\mathcal{C}_{0}}$ is trace-class on $\mathcal{H}_{s}$, we need to impose that $t>r s+\frac{1}{2}$. In this case, we know from Example 2 and Proposition 3 in Section 18 of [33] that the small balls asymptotic

$$
\log \left(\mu_{0}\left(B_{\epsilon}^{1}\left(a^{\dagger}\right)\right) \succsim-\epsilon^{-\rho}\right.
$$

is satisfied for $\mu_{0}$ with $\rho=\frac{-1}{t-r-1}$.

For this problem we adapt Theorem 3.4 by optimising over $s$ in the appropriate range as described in Remark 3. Moreover, Fernique's theorem [4] for Gaussian measures motivates us setting $e=2$ and $\rho=\frac{-1}{t-r-1}$ as discussed above.

Corollary 3.6. Let the prior and the observational noise be specified as in Equation (23) and (24). If the following optimisation problem has a solution $\kappa^{\star}>0$, then for any $\kappa<\kappa^{\star}$ the posterior $\mu^{y_{n}}$ is consistent in $\mathcal{H}^{1}$ for $a^{\dagger}$ in $\mathcal{H}^{s}$ with rate $n^{-\kappa}$.

Maximize $\kappa$ with respect to $\kappa, p \geq 1, \eta, \theta \geq 0,1+\frac{1}{2 r}<s<\frac{t-\frac{1}{2}}{r}$ subject to

$$
\begin{array}{ll}
\frac{1}{2}+\eta \frac{p}{q}-\kappa \lambda p & <1-2 \kappa \\
\frac{1}{2}-\eta+(1-\lambda) q \theta & <1-2 \kappa \\
\frac{1}{t-r-1} \kappa & <2 \theta \\
\frac{1}{t-r-1} \kappa & <1-2 \kappa \\
\left(\eta \frac{p}{q}-\frac{1}{2}\right) \lambda p & <-\kappa \\
\lambda p & <2 \\
(1-\lambda) q & <2 \\
\left(\frac{1}{2}-\eta\right)\left(1+\frac{1}{2-(1-\lambda) q}\right) & <\max (1-2 \kappa, \theta 2)
\end{array}
$$


where $\lambda:=\frac{s-1-\sigma_{0}}{s-1}$.

We now recast our problem reformulating it in the setting and notation of [30]. Letting $\zeta$ be $H$-valued white noise, our problem corresponds to recovering $a$ from

$$
y=a+\frac{1}{\sqrt{n}} \Gamma^{\frac{1}{2}} \zeta
$$

This problem is equivalent to

$$
\tilde{Y}=K a+\frac{1}{\sqrt{n}} \zeta
$$

where $K=\Gamma^{\frac{1}{2}}$. Let $\left\{f_{n}\right\}$ be an orthonormal basis of eigenvectors of $\Gamma$ on $H$. In order to adapt the notation of [30], we write $H_{2}:=H$ and note that $H_{1}$ will be equivalent to the Cameron-Martin space which takes the form

$$
H_{1}=S_{\mathcal{H}_{2}}^{r}:=\left\{v \in \mathcal{H}_{2} \mid v=\sum v_{i} f_{i} \text { s.t. } \sum v_{i}^{2} i^{2 r}<\infty\right\}
$$

with orthonormal basis $e_{k}=f_{k} / k^{r}$. Moreover, let $K: H_{1} \rightarrow H_{2}$ be defined as

$$
K e_{k}:=\Gamma^{-\frac{1}{2}} e_{k}=\frac{\lambda_{k}}{k^{r}} f_{k}
$$

In order to match Assumption 3.1 in [30], we have to bound the eigenvalues $\kappa_{i}$ of $K^{T} K$ as follows

$$
M^{-1} i^{-p} \leq \kappa_{i} \leq M i^{-p} .
$$

We determine these eigenvalues by noting that

$$
\left\langle K^{T} f_{k}, e_{j}\right\rangle_{H_{2}}=\left\langle f_{k}, K e_{j}\right\rangle_{H_{2}}=\delta_{j k} \frac{\lambda_{k}}{k^{r}} .
$$

The calculation above yields

$$
K^{T} K f_{k}=\left(\frac{\lambda_{k}}{k^{r}}\right)^{2} f_{k}
$$

and thus

$$
\kappa_{k}=\left(\frac{\lambda_{k}}{k^{r}}\right)^{2} \asymp 1=n^{0} \Rightarrow p=0 .
$$

As in Equation 25, we identify the covariance operator of $\mu_{0}$ on $H_{1}$ through its eigenvalues

$$
\tilde{\lambda}_{k} \asymp k^{-2 t+2 r} .
$$

By Theorem 4.1 in [30] the posterior contraction rate is given by

$$
n^{-\frac{\alpha \wedge \beta}{1+2 \alpha+2 p}}
$$

where $-1-2 \alpha=-2 t+2 r$ (compare Equation (3.5) in [30]) and $\beta$ is the regularity of the truth. As above, we suppose that $\beta \geq \alpha$ resulting in

$$
\kappa_{\mathrm{opt}}=\frac{t-r-\frac{1}{2}}{2(t-r)-1} .
$$




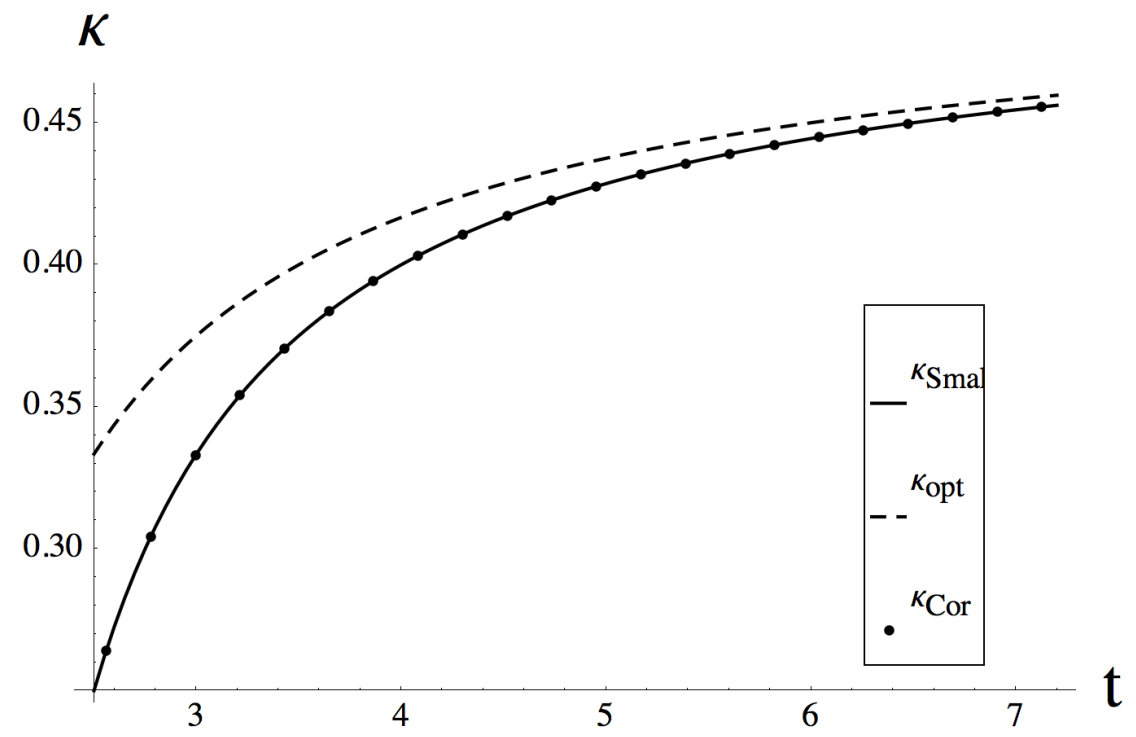

Figure 1. Posterior consistency rate for the Bayesian regression model with the noise and prior given in Equations 233 and 24. We denote the rate obtained in 30. and the one based on Corollary 3.6 as $\kappa_{\text {Opt }}$ and $\kappa_{\text {Cor }}$, respectively. We also plot $\kappa_{\text {Small Ball }=\frac{t-r-1}{2(t-r)-1}}$ an upper bound on the rate that is obtainable with our method which is based on the small ball asymptotics of the prior.

In Figure 1, we use numerical optimisation to compare our rate to the optimal one for $r=1$ with varying $t$.

Just considering Inequality (26) (essential to our approach since this implies that the Cameron-Martin term dominates the prior measure c.f. Equation (C.2) yields

$$
\kappa_{\text {Possible }}=\frac{t-r-1}{2(t-r)-1}
$$

which coincides with the rate $\kappa_{\text {Cor }}$ obtained by solving the optimisation problem in Corollary 3.6. Thus, even if we are able to improve our bounds, there is a genuine gap between our rate and the optimal rate in the case of Gaussian priors. The reason for this gap is that Theorem 3.4 is applicable to any prior satisfying the stated regularity and small ball assumptions. Nevertheless, Figure 1 indicates that the obtained rates are quite close. In contrast, [30] is only applicable to Gaussian priors for which the Gaussian stucture of the prior and the posterior are explicitly used.

\subsection{Pointwise Observations in the Large Data Limit}

We consider the following non-parametric Bayesian regression problem

$$
y_{i}=a\left(x_{i}\right)+\xi_{i} \quad i:=1, \ldots, n
$$

with $a: D \rightarrow \mathbb{R}, D$ a bounded domain and $\xi_{i} \stackrel{\text { i.i.d. }}{\sim} \mathcal{N}\left(0, \sigma^{2}\right)$. We assume that a prior $\mu_{0}$ is supported on $C(D, \mathbb{R})$ resulting in a posterior of the form

$$
\frac{d \mu^{y_{n}}}{d \mu_{0}} \propto \exp \left(-\sum_{i=1}^{n} \frac{\left(a\left(x_{i}\right)-y_{i}\right)^{2}}{2 \sigma^{2}}\right) \text {. }
$$


Subsequently, we will prove posterior consistency for this problem for the case $D=[0,1]$. However, the same reasoning applies to any bounded domain $D \subseteq \mathbb{R}^{d}$ but the actual posterior consistency rate depends on $d$.

As in the previous section, we suppose that the data $y_{i}$ in Equation 28 is generated for a fixed 'truth' $a^{\dagger}$. Hence

$$
\begin{aligned}
y_{i} & =a^{\dagger}\left(x_{i}\right)+\xi_{i} \\
\frac{d \mu^{y_{1: n}}}{d \mu_{0}} & \propto \exp \left(-\sum_{i=1}^{n} \frac{\left(a\left(x_{i}\right)-a^{\dagger}\left(x_{i}\right)\right)^{2}+2\left(a\left(x_{i}\right)-a^{\dagger}\left(x_{i}\right)\right) \xi_{i}}{2 \sigma^{2}}\right) .
\end{aligned}
$$

In this setup posterior consistency depends on the properties of the prior as well as on the sequence $\left\{x_{i}\right\}_{i \in \mathbb{N}}$. In the following, we discuss appropriate assumptions on both giving rise to Theorem 3.7. Moreover, we relate this result with its assumptions to the literature.

Assumption 4. There exist $\beta \in(0,1]$ and $L>0$ such that

$$
\|a\|_{\beta} \leq L \text { and }\|a\|_{\infty} \leq L \quad \mu_{0} \text {-a.s. }
$$

As $n$ increases, we gain more and more information about the function $a$. In particular, if $\left\{x_{i}\right\}_{i \in \mathbb{N}}$ is dense in $[0,1]$ it is even possible to reconstruct the value of $a^{\dagger}(x)$ from $y_{i}$. More precisely, let $x \in[0,1]$ be arbitrary, then there are $\left|x_{n_{j}}-x\right| \leq \frac{1}{j^{\frac{2}{\beta}}}$ such that

$$
a(x)=\lim _{J \rightarrow \infty} \frac{1}{J} \sum_{j=1}^{J} a\left(x_{n_{j}}\right) .
$$

However, we will see that this is not sufficient for posterior consistency. In fact, we will give an example of posterior inconsistency for this case. So far, the problem of posterior consistency for this type of regression problems has mainly been investigated for random evaluation points $x_{i}$ which are known as random covariates. Appropriate results of this type can be found in [39, 44]. An exception is [6] where posterior consistency without a rate with respect to the $L^{1}$-norm for deterministic $x_{i}$ is shown. This result is obtained under the following assumption.

Assumption 5. [6] Suppose that there exists a constant $K$ such that whenever $b-a \geq$ $\frac{1}{K n}$ for $0<a<b<1$ there is at least one $i$ such that $x_{i} \in(a, b)$.

The above condition guarantees that the number of observations in each interval satisfies a lower bound proportional to its size. More precisely, an interval of size $n^{-\kappa}$ has at least order $n^{1-\kappa}$ for $n$ being large enough. This can be seen by chopping the interval into intervals of size $\frac{1}{K n}$. In contrast to [6], we are also able to obtain a posterior consistency rate under this assumption. Posterior consistency without a rate can be concluded under the following weaker Assumption.

Assumption 6. We suppose that for $\left\{x_{i}\right\}_{i \in \mathbb{N}}$ there exists a $K>0$ such that for any $a<b \in[0,1]$ there is an $N(a, b)$ such that

$$
F_{n}(b)-F_{n}(a) \geq K(b-a) \quad \forall n>N(a, b)
$$

where $F_{n}$ denotes the empirical distribution of $\left\{x_{i}\right\}_{i=1}^{n}$. 
Theorem 3.7. Suppose that the Assumptions 4 and 6 are satisfied. Then $\mu^{y_{1: n}}$ is posterior consistent with respect to the $L^{\infty}{ }_{-n o r m}$ for any $a^{\dagger} \in \operatorname{supp}_{C^{\beta}}\left(\mu_{0}\right)$. Moreover, if Assumption 5 and the small ball asymptotic

$$
\log \left(\mu_{0}\left(B_{\epsilon}^{L^{\infty}}\left(a^{\dagger}\right)\right) \succsim-\epsilon^{-\rho}\right.
$$

are satisfied, then $\mu^{y_{1: n}}$ is posterior consistent with respect to the $L^{\infty}$-norm with any rate $n^{-\kappa}$ and

$$
\kappa<\min \left\{\frac{1}{2\left(2+\frac{1}{\beta}\right)}, \frac{2 \beta}{(2 \beta+1)(2+\rho)}\right\} .
$$

Proof. As in Theorem 3.3, posterior consistency is implied by

$$
\sup _{\left(\xi_{1}, \ldots, \xi_{n}\right) \in S_{n}} \frac{\mu^{y_{1: n}}\left(B_{\epsilon n^{-\kappa}}^{L^{\infty}}\left(a^{\dagger}\right)^{c}\right)}{\mu^{y_{1: n}}\left(B_{\epsilon n^{-\kappa}}^{L^{\infty}}\left(a^{\dagger}\right)\right)} \rightarrow 0 \text { for } n \rightarrow \infty
$$

for increasing sets $S_{n}$ such that $\mu_{\xi}\left(\left(\xi_{1}, \ldots, \xi_{n}\right) \in S_{n}\right) \rightarrow 1$. For notational convenience we write $h:=a-a^{\dagger}, S:=\sqrt{\sum_{i=1}^{n} h\left(x_{i}\right)^{2}}$ and we denote by $\eta$ a generic $\mathcal{N}\left(0, \sigma^{2}\right)$ random variable. This allows us to rewrite the posterior in Equation $(29)$ as

$$
\frac{d \mu^{y_{1: n}}}{d \mu_{0}} \propto Z(n, \eta) \exp \left(-\frac{1}{2 \sigma^{2}}\left(S^{2}+2 S \eta\right)\right) .
$$

Since $y_{1: n}$ is finite dimensional, it is easy to see that $Z(n, \eta)$ is bounded from above and below. Again, fixing $\gamma>0$, we only need to consider $\eta \in B_{n^{\gamma}}(0)$. Thus, for $0<l_{\epsilon}<1$ we have a lower bound on

$$
\mu^{y_{1: n}}\left(B_{\epsilon}^{L^{\infty}}\left(a^{\dagger}\right)\right) \geq Z(n, \eta) \exp \left(-n \frac{l_{\epsilon}^{2} \epsilon^{2}}{2 \sigma^{2}}-\frac{l_{\epsilon} \epsilon n^{\frac{1}{2}} n^{\gamma}}{\sigma^{2}}\right) \mu_{0}\left(B_{l_{\epsilon} \epsilon}^{L^{\infty}}\left(a^{\dagger}\right)\right) .
$$

In order to derive an upper bound on $\mu^{y_{1: n}}\left(B_{\epsilon}^{L^{\infty}}\left(a^{\dagger}\right)^{c}\right)$, let $a \in B_{\epsilon}^{L^{\infty}}\left(a^{\dagger}\right)^{c}$ be chosen arbitrarily and notice that $f(S)=-S^{2}+S n^{\gamma}$ is decreasing for $S>n^{\gamma}$. The upper bound on $\mu^{y_{1: n}}\left(B_{\epsilon}^{L^{\infty}}\left(a^{\dagger}\right)^{c}\right)$ therefore boils down to a lower bound on $S$ that is larger than $n^{\gamma}$. In fact, there is $\hat{x}$ such that $\left|a^{\dagger}(\hat{x})-a(\hat{x})\right| \geq \epsilon$. Applying Hölder continuity yields

$$
\left|a^{\dagger}(x)-a(x)\right| \geq \epsilon / 2 \text { for } x \in(\hat{x}-\Delta x, \hat{x}+\Delta x]
$$

for $\Delta x=\left(\frac{\epsilon}{4 L}\right)^{\frac{1}{\beta}}$. Let $I$ be the following index set

$$
I=\left\{i \mid x_{i} \in(\hat{x}-\Delta x, \hat{x}+\Delta x]\right\} .
$$

For $n$ larger than $N_{\epsilon}=\max \{N(i \Delta x,(i+1) \Delta x) \mid i=0 \ldots\lfloor 1 / \Delta x\rfloor-1\}$ it follows that

$$
K \frac{1}{2} \Delta x \leq F_{n}(\hat{x}+\Delta x)-F_{n}(\hat{x}-\Delta x)=\frac{|I|}{n} .
$$

If we only consider $x_{i}$ with $i \in I$, we obtain that

$$
S \geq \sqrt{\frac{\epsilon^{2}}{4} n K \frac{\Delta x}{2}}
$$


which gives rise to the following upper bound

$$
\mu^{y_{1: n}}\left(B_{\epsilon}^{L^{\infty}}\left(a^{\dagger}\right)^{c}\right) \leq Z(n, \xi) \exp \left[-\frac{n \epsilon^{2} K \Delta x}{8 \sigma^{2}}+\left(\frac{K}{2} \Delta x\right)^{\frac{1}{2}} \frac{\epsilon}{4 \sigma^{2}} n^{\frac{1}{2}+\gamma}\right] .
$$

By choosing $l_{\epsilon}$ small enough, we also know that

$$
\frac{\mu^{y_{1: n}}\left(B_{\epsilon}^{L^{\infty}}\left(a^{\dagger}\right)^{c}\right)}{\mu^{y_{1: n}}\left(B_{\epsilon}^{L^{\infty}}\left(a^{\dagger}\right)\right)} \rightarrow 0 \quad \text { as } n \rightarrow \infty .
$$

In order to obtain a rate of posterior consistency, we use $\tilde{\kappa}>\kappa$ and hence

$$
\mu^{y_{n}}\left(B_{n^{-\kappa}}^{L^{\infty}}\left(a^{\dagger}\right)\right) \geq \mu^{y_{n}}\left(B_{n^{-\tilde{\kappa}}}^{L^{\infty}}\left(a^{\dagger}\right)\right) \geq Z(n, \xi) \exp \left[-\frac{1}{2 \sigma^{2}} n^{1-2 \tilde{\kappa}}-\frac{1}{2 \sigma^{2}} n^{\frac{1-\tilde{\kappa}}{2}+\gamma}-c n^{\tilde{\kappa} \rho}\right]
$$

Thus, Equation (31) implies that

$$
\mu^{y_{1: n}}\left(B_{n^{-\kappa}}^{L^{\infty}}\left(a^{\dagger}\right)^{c}\right) \leq Z(n, \xi) \exp \left(-\frac{K}{8 \sigma^{2}(4 L)^{\frac{1}{\beta}}} n^{1-\left(2+\frac{1}{\beta}\right) \kappa}+\frac{K^{\frac{1}{2}} n^{\frac{1}{2}-\kappa\left(1+\frac{1}{2 \beta}\right)}}{2 \sigma^{2} \sqrt{2}(4 L)^{\frac{1}{\beta}}}\right) .
$$

The first term in the exponential in Equation 32 is dominant over the corresponding term in Equation (33) by choosing

$$
\tilde{\kappa}:=\kappa\left(1+\frac{1}{2 \beta}\right)+\gamma .
$$

Moreover, the first term in the Equations 32 and (33) is dominant over the other terms respectively if

$$
\begin{array}{ll}
1-2 \tilde{\kappa} & >\tilde{\kappa} \rho \\
1-2 \tilde{\kappa} & >\frac{1-\tilde{\kappa}}{2}+\gamma \\
1-\left(2+\frac{1}{\beta}\right) \kappa & >\frac{1}{2}-\kappa\left(1+\frac{1}{2 \beta}\right) .
\end{array}
$$

These three inequalities are respectively implied by

$$
\begin{array}{ll}
\frac{1-\rho \gamma-2 \gamma}{\left(1+\frac{1}{2 \beta}\right)(2+\rho)} & >\kappa \\
\frac{2-10 \gamma}{6\left(1+\frac{1}{2 \beta}\right)} & >\kappa \\
\frac{1}{2+\frac{1}{\beta}} & >\kappa .
\end{array}
$$

Choosing $\gamma$ small enough, we see that $\mu^{y_{n}}$ is consistent in $L^{\infty}$ with any rate

$$
\kappa<\min \left\{\frac{1}{3\left(1+\frac{1}{2 \beta}\right)}, \frac{1}{\left(1+\frac{1}{2 \beta}\right)(2+\rho)}\right\} \text {. }
$$

The assumptions in the theorem above can be justified because a slight violation leads to the example of posterior inconsistency in the next section. 
3.2.1. Example of Posterior Inconsistency In this section, we construct a counterexample to illustrate that despite the strong Assumption 4 it is not sufficient for $\left\{x_{i}\right\}_{i=1}^{n}$ to be dense in order to establish posterior consistency. Given such a sequence it is always possible to extract a subsequence satisfying Assumption 6. Even though all the other observations can be viewed as additional, we will choose a prior so that the posterior sequence is not consistent.

In the following, we choose the prior concentrated on functions $g$ which are continuous, satisfy $g\left(\frac{1}{2}\right)=0$ and are linear on $\left[0, \frac{1}{2}\right]$ and $\left[\frac{1}{2}, 1\right]$. By identifying $g(0)$ and $g(1)$ with the first and second component respectively the following two-dimensional example can be extended to the setting of Equation (28). This extension is an example of posterior inconsistency with respect to the $L^{p}$-norm for $1 \leq p \leq \infty$ because any of these norms is equivalent to $\|(g(0), g(1))\|$ for an arbitrary norm on $\mathbb{R}^{2}$.

Example 1. We consider the following prior on $\mathbb{R}^{2}$

$$
\mu_{0}=M \sum_{k=1}^{\infty} \delta_{\left(\frac{1}{\sqrt{k}}, 0\right)} \exp \left(-2 k^{2}\right)+\delta_{\left(\frac{1}{2 \sqrt{k}}, 1\right)} \exp \left(-k^{2}\right)
$$

and we choose $a^{\dagger}=(0,0)$ as 'truth'. The data consists of $n$ and $n^{\theta}$ with $0<\theta<1$ being measurements of the form

$$
y_{i}=a_{1}^{\dagger}+\xi_{i} \text { and } \tilde{y}_{i}=a_{2}^{\dagger}+\tilde{\xi}_{i} \text { with } \xi_{i}, \tilde{\xi}_{i} \stackrel{i . i . d .}{\sim} \mathcal{N}(0,1),
$$

respectively. Consequently, the posterior takes the form

$$
\mu^{\left(y_{1: n}, \tilde{y}_{1: n^{\theta}}\right)} \propto \mu_{0}\left(d a_{1}, d a_{2}\right) \exp \left(-\frac{1}{2} \sum_{j=1}^{n}\left(a_{1}-\xi_{j}\right)^{2}-\frac{1}{2} \sum_{j=1}^{n^{\theta}}\left(a_{2}-\tilde{\xi}_{j}\right)^{2}\right) .
$$

Here, posterior consistency of $\mu^{\left(y_{1: n}, \tilde{y}_{1: n^{\theta}}\right)}$ is equivalent to the statement that for any $K$ there is $l_{n} \uparrow 1$ such that

$$
\mathbb{P}_{\eta}\left(\mu^{\left(y_{1: n}, \tilde{y}_{1: n} \theta\right)}\left(\bigcup_{k=K}^{\infty}\left(\frac{1}{\sqrt{k}}, 0\right)\right) \geq l_{n}\right) \rightarrow 1 \text { for } n \rightarrow \infty .
$$

We will not only show that $\mu^{\left(y_{1: n}, \tilde{y}_{1: n^{\theta}}\right)}$ is posterior inconsistent but also that there is $l_{n} \downarrow 0$ such that for $A=\bigcup_{k=1}^{\infty}\left(\frac{1}{k}, 0\right)$

$$
\mathbb{P}_{\eta}\left(\mu^{\left(y_{1: n}, \tilde{y}_{1: n} \theta\right)}(A) \leq l_{n}\right) \rightarrow 1 \text { for } n \rightarrow \infty .
$$

Because of $\mu^{\left(y_{1: n}, \tilde{y}_{1: n} \theta\right)}(A)+\mu^{\left(y_{1: n}, \tilde{y}_{1: n^{\theta}}\right)}\left(A^{c}\right)=1$, we may proceed as in the proofs of the Theorems 3.3 and 3.7 and thus it is enough to construct sets of increasing $\mathbb{P}_{\xi}$-probability such that on these sets

$$
\begin{aligned}
\frac{\mu^{\left(y_{1: n}, \tilde{y}_{1: n^{\theta}}\right)}(A)}{\mu^{\left(y_{1: n}, \tilde{y}_{1: n^{\theta}}\right)}\left(A^{c}\right)} & =\frac{\sum_{k} \exp \left(-\frac{1}{2} \sum_{j=1}^{n}\left(\frac{1}{\sqrt{k}}-\xi_{j}\right)^{2}-\frac{1}{2} \sum_{j=1}^{n^{\theta}} \tilde{\xi}^{2}-2 k^{2}\right)}{\sum_{k} \exp \left(-\frac{1}{2} \sum_{j=1}^{n}\left(\frac{1}{2 \sqrt{k}}-\xi_{j}\right)^{2}-\frac{1}{2} \sum_{j=1}^{n^{\theta}}\left(\tilde{\xi}_{j}-1\right)^{2}-k^{2}\right)} \\
& =\frac{\sum_{k} \exp \left(-\frac{1}{2} \sum_{j=1}^{n} \frac{1}{k}-\frac{2}{k} \xi_{j}-2 k^{2}\right)}{\sum_{k} \exp \left(-\frac{1}{2} \sum_{j=1}^{n} \frac{1}{4 k}-\frac{1}{\sqrt{k}} \xi_{j}-\frac{1}{2} \sum_{j=1}^{n^{\theta}} 1-2 \tilde{\xi}_{k}-k^{2}\right)} \rightarrow 0 .
\end{aligned}
$$


The $\mathbb{P}_{\xi}$-probabilities of $\left|\sum_{j=1}^{n} \xi_{j}\right|>M n^{\frac{1}{2}+\gamma}$ and $\left|\sum_{j=1}^{n^{\theta}} \tilde{\xi}_{j}\right|>M n^{\frac{1}{2}+\gamma}$ are exponentially small in $n$. Thus, it is enough to consider

$$
\begin{aligned}
\frac{\sum_{k} \exp \left(-\frac{1}{2} n \frac{1}{k}-2 k^{2}\right)}{\sum_{k} \exp \left(-\frac{1}{2} n \frac{1}{4 k}-k^{2}\right)} & =\frac{\sum_{k}^{\sqrt{n}} \exp \left(-\frac{1}{2} n \frac{1}{k}-2 k^{2}\right)+\sum_{\sqrt{n}}^{\infty} \exp \left(-\frac{1}{2} n \frac{1}{k}-2 k^{2}\right)}{\sum_{k}^{\sqrt{n}} \exp \left(-\frac{1}{2} n \frac{1}{4 k}-k^{2}\right)+\sum_{\sqrt{n}}^{\infty} \exp \left(-\frac{1}{2} n \frac{1}{4 k}-k^{2}\right)} \\
& \leq \max \left\{\exp \left(-\frac{3}{4} \sqrt{n}\right), \exp (-n)\right\} \rightarrow 0 \text { as } n \rightarrow \infty
\end{aligned}
$$

Hence we have shown that $\mu^{y_{n}}$ is not posterior consistent.

This example relies on the prior having strong correlations between its two components. Therefore it seems an interesting question how the assumptions on $\mu_{0}$ can be strengthened in order to relax those on $\left\{x_{i}\right\}$.

\subsection{Convergence in Stronger Norms}

We conclude this section by showing that interpolation inequalities can be used in order to strengthen the norm in which the posterior concentrates. In particular we consider the small noise limit as described in Section 3.1.

Suppose we know that the posterior concentrates around the truth $a^{\dagger}$ in the Cameron-Martin norm $\|\cdot\|_{1}$. In order to show consistency in $\|\cdot\|_{r}$, we write

$$
\begin{aligned}
\left\{\left\|a-a^{\dagger}\right\|_{r}>\epsilon\right\} & \subset\left\{\left\|a-a^{\dagger}\right\|_{1}^{\lambda}\left\|a-a^{\dagger}\right\|_{s}^{1-\lambda}>\epsilon\right\} \\
& \subset\left\{\left\|a-a^{\dagger}\right\|_{1}^{\lambda}>\frac{\epsilon}{K}\right\} \cup\left\{\left\|a-a^{\dagger}\right\|_{s}^{1-\lambda}>K\right\} .
\end{aligned}
$$

The posterior probability of the first set is small due to the posterior consistency in $\mathcal{H}^{1}$. The posterior probability of the second set is small due to the tails of the prior and the posterior. Obtaining estimates of this type can be done similarly to the steps subsequent to Equation (C.12) in the proof of Theorem 3.4. Using this technique, it is also possible to apply the results of this section to the EIP in the next section. A similar technique based on interpolation inequalities between Hlder spaces applies to the large data limit and is also used for the EIP.

\section{Posterior Consistency for an Elliptic Inverse Problem}

In Section 2.3. we introduced the idea of reducing posterior consistency of the EIP to that of the BRP. For this example we demonstrate our method for both the small noise and the large data limit. We start by giving the proof for the small noise limit in detail before sketching the same steps for the large data limit. We emphasise the case of posterior consistency in the small noise limit because of its analogy with convergence results for regularisation methods.

\subsection{Posterior Consistency in the Small Noise Limit}

Using Theorem 3.3 from Section 3 to conclude posterior consistency of the EIP (c.f. Section 2.3) is not entirely straightforward because we have to lift the posterior 
consistency for the BRP to $C^{2}$. Moreover, we have to find appropriate assumptions on the prior $\mu_{0}$ so that the push forward prior $p_{\star} \mu_{0}$ satisfies the assumptions of the Theorem 3.3. Again, a rate of posterior consistency is obtained if the prior satisfies appropriate small ball asymptotics. In a second step we verify those for the so-called uniform priors which are based on a series expansion with uniformly distributed coefficients, for details see below or consider [38, 29, 41].

In order to formulate assumptions on $\mu_{0}$ implying that $p_{\star} \mu_{0}$ satisfies the assumptions of Theorem 3.3, we assume for simplicity that $\xi \sim \mathcal{N}\left(0,\left(-\Delta_{\text {Dirichlet }}\right)^{-r}\right)$ where $\Delta_{\text {Dirichlet }}$ denotes the Laplacian with homogeneous Dirichlet conditions. In this case the abstract Hilbert scale $\mathcal{H}^{s}$ (c.f. Appendix A corresponds to the standard Sobolev space $H^{r s}$. Thus, the almost sure bounds in Theorem 3.3 are implied by the appropriate assumptions on the prior and classical results from [18, 24].

Moreover, the choice $\xi \sim \mathcal{N}\left(0,\left(-\Delta_{\text {Dirichlet }}\right)^{-r}\right)$ also implies that Assumption 3 holds for $\sigma_{0}=\frac{d}{2 r}$. This is due to the fact that the operator $\left(-\Delta_{\text {Dirichlet }}\right)^{-r}$ has eigenvalues $\lambda_{k}^{2}$ with $\lambda_{k} \asymp k^{-2 r / d}$ (see Section Appendix A for notation) where $d$ denotes the dimension of the domain $D$. These results are called Weyl asymptotics and further details can be found in [45] and 37].

The following theorem summarises the consequences for the posterior consistency of the EIP.

Theorem 4.1. Suppose that the noise is given by $\xi \sim \mathcal{N}\left(0,\left(-\Delta_{\text {Dirichlet }}\right)^{-r}\right)$ and that the prior $\mu_{0}$ satisfies

$$
a(x) \geq \lambda>0 \forall x \in D \text { and }\|a\|_{C^{\alpha}} \leq \Lambda \quad \text { for } \mu_{0} \text {-a.e. } a \text { and for } \alpha>1 .
$$

If $\alpha>r+\frac{d}{2}-2, \beta+1>r$ and $a^{\dagger} \in \operatorname{supp}_{C^{\beta}} \mu_{0}$, then the EIP is posterior consistent with respect to the $C^{\tilde{\alpha}}$-norm for any $\tilde{\alpha}<\alpha$. Additionally, if

$$
\log \left(\mu_{0}\left(B_{\epsilon}^{C^{\beta}}\left(a^{\dagger}\right)\right) \succsim-\epsilon^{-\rho}\right.
$$

then the EIP is posterior consistent with respect to the $L^{\infty}$-norm with rate $n^{-\kappa}$ for any $\kappa$ such that

$$
\kappa<\left(\frac{\alpha}{\alpha+2+\frac{d}{2}-r} \wedge 1\right)\left(\frac{1}{2+\rho} \wedge \frac{\alpha}{2\left(\alpha+1+\frac{d}{2 r}\right)}\right) .
$$

Before proving Theorem 4.1, we notice that forward stability results (as Proposition 2.1) can be used to transfer small ball asymptotics from $\mu_{0}$ to $\tilde{\mu}_{0}=p_{\star} \mu_{0}$.

Lemma 4.2. If the prior satisfies the small ball asymptotic

$$
\log \left(\mu_{0}\left(B_{\epsilon}^{C^{\beta}}\left(a^{\dagger}\right)\right) \succsim-\epsilon^{-\rho}\right.
$$

then

$$
\log \left(\tilde{\mu_{0}}\left(B_{\epsilon}^{C^{\beta+1}}\left(p^{\dagger}\right)\right) \succsim-\epsilon^{-\rho}\right.
$$


Proof. Proposition 2.1 implies that

$$
\begin{array}{ll}
\left\|a-a^{\dagger}\right\|_{C^{\beta}} & \leq \epsilon \Rightarrow\left\|p-p^{\dagger}\right\|_{C^{\beta}} \leq M \epsilon \\
\text { and } p\left(B_{\epsilon}^{C^{\beta}}\left(a^{\dagger}\right)\right) \subseteq B_{C \epsilon}^{C^{\beta+1}}\left(p^{\dagger}\right) .
\end{array}
$$

Hence the statement follows.

Having established Lemma 4.2, we are now in the position to prove the main theorem of this section.

Proof of Theorem 4.1. Subsequently, $M$ will denote a generic constant in different contexts that may change form line to line. We will first prove posterior consistency in $L^{\infty}$ before we use an interpolation inequality to bootstrap it to $C^{\tilde{\alpha}}$. In order to prove posterior consistency in the $L^{\infty}$-norm, it is enough to show posterior consistency of the BRP in the $C^{2}$-norm because

$$
\mu^{y_{n}}\left(B_{\epsilon}^{L^{\infty}}\left(a^{\dagger}\right)\right)=\tilde{\mu}^{y_{n}}\left(p\left(B_{\epsilon}^{L^{\infty}}\left(a^{\dagger}\right)\right) \geq \tilde{\mu}^{y_{n}}\left(B_{\frac{\epsilon}{M}}^{C^{2}}\left(p^{\dagger}\right)\right)\right.
$$

which follows by an application of Proposition 1 and a change of variables (see Theorem Appendix B.1). Using Theorem 6.19 from [24], we may conclude that

$$
\|p\|_{H^{\alpha+2}} \lesssim\|p\|_{C^{\alpha+2}} \leq K \tilde{\mu}_{0^{-}} \text {a.s.. }
$$

Since $\alpha+2>r, p$ is $\tilde{\mu}_{0}$-a.s. an element of the Cameron-Martin space of $\mu_{\xi}$ as it corresponds to $H^{r}$. Posterior consistency of the BRP with respect to the $H^{r}$-norm is now implied by Theorem 3.3. Its conditions are satisfied because

$$
\|p\|_{\mathcal{H}^{s}} \leq M \Lambda \quad \tilde{\mu}_{0} \text {-a.s. }
$$

with

$$
s=\frac{\alpha+2}{r}>1+\frac{d}{2 r}=1+\sigma_{0} .
$$

Furthermore, Proposition 2.1 and the fact that $a^{\dagger} \in \operatorname{supp}_{C^{\beta}} \mu_{0}$ imply that $p\left(a^{\dagger}\right) \in$ $\operatorname{supp}_{H^{r}} \tilde{\mu_{0}}$. In order to bootstrap to posterior consistency in the $C^{2}$-norm, we use a generalisation of the Sobolev embedding theorem for Besov spaces and an interpolation inequality between Besov spaces on domains (for details consult [42]). We first note that $B_{22}^{\tau}=H^{\tau}$ and $C^{\tau}=B_{\infty \infty}^{\tau}$ for $\tau \notin \mathbb{Z}$. In particular Theorem 4.33 in [42] implies that

$$
\|g\|_{B_{\infty \infty}^{r-\frac{d}{2}-\gamma}} \leq M\|g\|_{H^{r}}
$$

for $\gamma>0$ being small. If $r>\frac{d}{2}+2$, we can conclude posterior consistency in the $C^{2}$-norm because

$$
B_{\frac{\epsilon}{M}}^{C^{2}}\left(p^{\dagger}\right) \supseteq\left\{p \mid\left\|p-p^{\dagger}\right\|_{B_{\infty \infty}^{r-\frac{d}{2}-\gamma}} \leq \frac{\epsilon}{M}\right\} \supseteq\left\{p \mid\left\|p-p^{\dagger}\right\|_{H^{r}} \leq \frac{\epsilon}{M}\right\}
$$


holds for $\gamma$ small enough. Otherwise, we use the interpolation inequality between Besov spaces subject of Theorem 4.17 in 42 ]

$$
\|g\|_{C^{2+\gamma}} \leq\|g\|_{B_{\infty \infty}^{r-\frac{d}{2}-\gamma}}^{\theta}\|g\|_{B_{\infty \infty}^{\alpha+2}}^{1-\theta}
$$

for $\gamma$ small enough and with $\theta=\frac{\alpha}{\alpha+2+\frac{d}{2}-r+\gamma}$. Similar to Equation (35), it follows that

$$
B_{\frac{\epsilon}{M}}^{C^{2}}\left(p^{\dagger}\right) \supseteq\left\{p \mid\left\|p-p^{\dagger}\right\|_{B_{\infty \infty}^{r-\frac{d}{2}-\gamma}}^{\theta} \leq \frac{\epsilon}{K M}\right\} \supseteq\left\{p \mid\left\|p-p^{\dagger}\right\|_{H^{r}} \leq \frac{\epsilon^{\theta^{-1}}}{M}\right\} .
$$

The Equations (35) and (37) allow us to bootstrap the posterior consistency of $\tilde{\mu}^{y_{n}}$ to $C^{2}$. Equation (34) implies posterior consistency of $\mu^{y_{n}}$ in the $L^{\infty}$-norm. Similarly, we bootstrap to posterior consistency in $C^{\tilde{\alpha}}$ for $\tilde{\alpha}<\alpha$ using the same interpolation technique as above.

In order to obtain a rate for posterior consistency, we first note that $\log \left(\mu_{0}\left(B_{\epsilon}^{C^{\beta}}\left(a^{\dagger}\right)\right) \succsim-\epsilon^{-\rho}\right.$ implies

$$
\log \left(\tilde{\mu}_{0}\left(B_{\epsilon}^{r}\left(a^{\dagger}\right)\right) \succsim-\epsilon^{-\rho}\right.
$$

due to Lemma 4.2. Now Theorem 3.3 implies posterior consistency of the sequence of posteriors $\tilde{\mu}^{y_{n}}$ in $H^{r}$ with any rate $\kappa$ such that

$$
\kappa<\frac{1}{2+\rho} \wedge \frac{\alpha+1}{2\left(\alpha+1+\frac{d}{2 r}\right)} .
$$

Using the interpolation inequality as above gives rise to posterior consistency for $\tilde{\mu}^{y_{n}}$ in $C^{2+\alpha}$ with rate $n^{-\kappa}$ for any $\kappa$ such that

$$
\kappa<\left(\frac{\alpha}{\alpha+2+\frac{d}{2}-r} \wedge 1\right)\left(\frac{1}{2+\rho} \wedge \frac{\alpha}{2\left(\alpha+1+\frac{d}{2 r}\right)}\right) .
$$

As above, this implies the same rate of posterior consistency for $\mu^{y_{n}}$ in $L^{\infty}$.

4.1.1. Uniform Prior In this section, we establish a rate of posterior consistency for the EIP with the so-called uniform prior introduced in [41, 29, 38]. This choice of the prior was motivated by the preceding analysis in the uncertainty quantification literature, see for instance [7, 8]. It is given by

$$
\mu_{0}=\mathcal{L}\left(a_{0}(x)+\sum_{i=1}^{\infty} \gamma_{i} z_{i} \psi_{i}(x)\right) \quad z_{i} \stackrel{\text { i.i.d. }}{\sim} \mathcal{U}[-1,1]
$$

where $\mathcal{L}$ denotes the law of a random variable. Moreover, we suppose that $\left\|\psi_{i}(x)\right\|_{C^{\beta}}=$ $1, \gamma_{i}>0$ and $S=\sum_{i=1}^{\infty} \gamma_{i}<\infty$ such that

$$
0<a_{\min } \leq a \leq a_{\max } \quad \mu_{0} \text {-a.s.. }
$$

In order to obtain a rate for the EIP with this prior, we derive a small ball asymptotic under an appropriate assumption on the decay of $\left\{\gamma_{i}\right\}$. 
Assumption 7. There exists $\nu^{\star} \in(0,1)$ such that for all $\nu>\nu^{\star}$

$$
S_{\nu}=\left(\sum_{i=1}^{\infty} \gamma_{i}^{\nu}\right)^{\frac{1}{\nu}}<\infty .
$$

Since the series in Equation (37) is absolutely convergent, we assume without loss of generality that $\gamma_{i}$ is decreasing. This allows us to use the following classical inequality from approximation theory [13]

$$
\left(\sum_{n>N} \gamma_{n}\right) \leq N^{1-\frac{1}{\nu}} S_{\nu} .
$$

Lemma 4.3. Suppose that $\mu_{0}$ is given as in Equation (37), Assumption 7 is satisfied with $\nu^{\star}$ and

$$
a^{\dagger}=\sum_{i=1}^{\infty} \gamma_{i} z_{i}^{\dagger} \psi_{i}(x) \quad \text { where } z_{i}^{\dagger} \in[-1,1] .
$$

Then for any $\nu>\nu^{\star}$

$$
\log \mu_{0}\left(B_{\epsilon}^{C^{\beta}}\left(a^{\dagger}\right)\right) \gtrsim-\epsilon^{-\frac{1}{\nu} \frac{1}{\nu-1}} .
$$

Proof. We obtain an asymptotic lower bound on the small ball probability by choosing an appropriate subset $D_{\epsilon}\left(a^{\dagger}\right)$ of $B_{\epsilon}^{C^{\beta}}\left(a^{\dagger}\right)$. We denote a generic element of this set by

$$
a=\sum_{i=1}^{\infty} \gamma_{i} z_{i} \psi_{i}(x)
$$

Choosing $N_{\epsilon}$ such that $\sum_{i=N_{\epsilon}}^{\infty} \gamma_{i} \leq \frac{\epsilon}{2}$, the corresponding terms contribute at most $\frac{\epsilon}{2}$ to the difference $\left\|a^{\dagger}-a\right\|$. The subset $D_{\epsilon}\left(a^{\dagger}\right)$ prescribes intervals for $z_{i} i=1 \ldots N_{\epsilon}$ such that this contribution is at most $\frac{\epsilon}{2}$, too. More precisely, let $\nu^{\star}<\tilde{\nu}<\nu$, then Equation (38) implies

$$
\sum_{n>N_{\epsilon}} \gamma_{n} \leq N_{\epsilon}^{1-\frac{1}{\nu}} S_{\tilde{\nu}} \leq \frac{\epsilon}{2}
$$

for $N_{\epsilon} \geq\left(\frac{2 S_{\tilde{\nu}}}{\epsilon}\right)^{\frac{1}{\nu}-1}$. Let the subset $D_{\epsilon}\left(a^{\dagger}\right) \subseteq B_{\epsilon}^{C^{\beta}}\left(a^{\dagger}\right)$ be given by

$$
\left\{a \mid\left(z_{i}^{\dagger}>0 \wedge z_{i}^{\dagger}-\frac{\epsilon}{2 S} \leq z_{i} \leq z_{i}^{\dagger}\right) \vee\left(z_{i}^{\dagger} \leq 0 \wedge z_{i}^{\dagger}+\frac{\epsilon}{2 S} \geq z_{i} \geq z_{i}^{\dagger}\right) 1 \leq i \leq N_{\epsilon}\right\} .
$$

Then

$$
\begin{aligned}
& \mu_{0}\left(B_{\epsilon}^{C^{\beta}}\left(a^{\dagger}\right)\right) \geq\left(\frac{\epsilon}{2 S}\right)^{N_{\epsilon}} \\
& \log \mu_{0}\left(B_{\epsilon}^{C^{\beta}}\left(a^{\dagger}\right)\right) \gtrsim N_{\epsilon} \log \epsilon \gtrsim-\epsilon^{-\frac{1}{\nu-1}} .
\end{aligned}
$$

Combining Lemma 4.3 and Theorem 4.1 results in the following theorem which characertises posterior consistency for this class of priors. 
Theorem 4.4. Let the prior $\mu_{0}$ be defined as in Equation (37) and let Assumption 7 be satisfied. Additionally, we assume that $\alpha \geq \beta \geq r+1, \alpha>r+\frac{d}{2}-2$ and $\|a\|_{\alpha} \leq K \mu_{0}-$ a.s.. Then the posterior $\mu^{y_{n}}$ is consistent for any

$$
a^{\dagger}=\sum_{i=1}^{\infty} \gamma_{i} z_{i}^{\dagger} \psi_{i}(x) \quad \text { where } z_{i}^{\dagger} \in[-1,1]
$$

with respect to the $L^{\infty}$-norm with rate $\epsilon_{n}=M(\kappa) n^{-\kappa}$ for any $\kappa$ such that

$$
\kappa<\left(\frac{\alpha}{\alpha+2+\frac{d}{2}-r} \wedge 1\right)\left(\frac{1-\nu}{2-\nu} \wedge \frac{\alpha-r+2}{2 \alpha+d-2 r+4}\right) \text {. }
$$

\subsection{Posterior Consistency in the Large Data Limit}

In the following we show that the results for the BRP can be transferred to posterior consistency results in the large data limit for the EIP. We consider only the case $d=1$ with $D=[0,1]$ as the general case is similar. Furthermore, assuming that the observations are of the form

$$
y_{i}=p\left(x_{i} ; a\right)+\xi \quad i=1 \ldots n
$$

the sequence of posteriors is given by

$$
\frac{d \mu^{y_{n}}}{d \mu_{0}}(a) \propto \exp \left(-\sum_{i=1}^{n} \frac{\left(p(a)\left(x_{i}\right)-y_{i}\right)^{2}}{2 \sigma^{2}}\right) .
$$

Posterior consistency of the EIP in $L^{\infty}$ can then be derived on the basis of Theorem 3.7 .

Theorem 4.5. Suppose that the sequence $\left\{x_{i}\right\}$ satisfies Assumption 6 , $\|a\|_{C^{\gamma}} \leq L \mu_{0^{-}}$ a.s. with $\gamma>1$ and $a \geq a_{\text {min }} \mu_{0}$-a.s.. If $a^{\dagger} \in \operatorname{supp}_{C} \gamma \mu_{0}$, then the EIP is posterior consistent in the large data limit with respect to $C^{\tilde{\gamma}}$ for any $\tilde{\gamma}<\gamma$.

Proof. An application of Theorem 6.13 in [24] yields the existence of $M(D, \gamma)$ so that for all $a$ satisfying

$$
\|a\|_{C^{\gamma}} \leq S \text { and } a \geq a_{\min }
$$

there is a unique solution $p$ such that $\|p\|_{C^{2+\gamma}} \leq M$. Thus, $\tilde{\mu}_{0}=p_{\star} \mu_{0}$ satisfies the assumptions of Theorem 3.7 implying that $\tilde{\mu}^{y_{1: n}}$ is posterior consistent in $L^{\infty}$. Using the interpolation inequality between $L^{\infty}$ and $C^{2+\gamma}$, we also obtain consistency in $C^{2}$. As in Theorem 4.1. Proposition 1 can be used in order to conclude posterior consistency of $\mu^{y_{1: n}}$ in $L^{\infty}$. We can bootstrap from $L^{\infty}(D)$ to $C^{\tilde{\gamma}}$ by interpolating between $L^{\infty}$ and $C^{\gamma}$. 


\section{Concluding Remarks}

In this article, we have established a novel link between stability results for an inverse problem and posterior consistency for the Bayesian approach to it. We have explicitly shown this link for an elliptic inverse problem (c.f. EIP) but the same method is also applicable for the general case. An instance is electrical impedance tomography (Caldern problem) for which stability results are available [3]. This example would lead to a very slow posterior consistency rate since its stability results are weak. Essentially, we would have to redo all the calculations on a log-scale instead of an algebraic scale.

So far, we need exponential moments of the prior for the Bayesian regression of functional response and for pointwise observations (see also Section 4.2.2 in [6]). For this reason it is harder to prove posterior consistency for example for log-Gaussian priors. Log-Gaussian measures have moments of arbitrary order but no exponential moments. This is a problem that we would like to pursue further in the future.

\section{Appendix A. Notation and Review of Technical Tools}

\section{Appendix A.1. Asymptotic Inequalities}

We use the following notation for asymptotic inequalities:

Let $a_{n}$ and $b_{n}$ be sequences in $\mathbb{R}$. We denote by $\mathbb{R} a_{n} \lesssim b_{n}$ that there are $N \in \mathbb{N}$ and $M \in \mathbb{R}$ such that $a_{n} \leq M b_{n}$ for $n \geq N$. Moreover, if $a_{n} \lesssim b_{n} \lesssim a_{n}$, we write $a_{n} \asymp b_{n}$.

Appendix A.1.1. Hilbert Scales In order to measure the smoothness of the noise and samples of the prior, we introduce Hilbert scales following [17]. Let $\Gamma$ be a self-adjoint, positive-definite, trace-class linear operator with eigensystem $\left(\lambda_{k}^{2}, \phi_{k}\right)$. We know that $\Gamma^{-1}$ is a densely defined, unbounded, symmetric and positive-definite operator because

$$
H=\overline{\mathcal{R}(\Gamma)} \oplus \operatorname{Ker}(\Gamma)^{\perp}=\overline{\mathcal{R}(\Gamma)} .
$$

We define the Hilbert scale by $\left(\left(\mathcal{H}^{t},\langle\cdot, \cdot\rangle_{t}\right)\right)_{t \in \mathbb{R}}$ with $\mathcal{H}^{t}:=\overline{\mathcal{M}}^{\|\cdot\|_{t}}$ for

$$
\begin{aligned}
\mathcal{M} & :=\bigcap_{n=0}^{\infty} \mathcal{D}\left(\Gamma^{-n}\right) \\
\langle u, v\rangle_{t} & :=\left\langle\Gamma^{-\frac{t}{2}} u, \Gamma^{-\frac{t}{2}} v\right\rangle \\
\|u\|_{t} & :=\left\|\Gamma^{-\frac{t}{2}} u\right\| .
\end{aligned}
$$

We will denote balls with respect to the $\|\cdot\|_{t}$-norm by

$$
B_{R}^{t}(u)=\left\{x \mid\|u-x\|_{t} \leq R\right\} .
$$

Moreover, these collection of norms satisfies an interpolation inequality

Proposition Appendix A.1. (Proposition 8.19 in [17]) Let $q<r<s$ then the following interpolation inequality holds

$$
\|x\|_{r} \leq\|x\|_{q}^{\frac{s-r}{s-q}}\|x\|_{s}^{\frac{r-q}{s-q}}
$$


Remark. Our definition here is slightly different from the literature in order to match it to the Sobolev spaces for $\Gamma=\left(-\Delta_{\text {Dirichlet }}\right)^{-1}$

\section{Appendix A.2. Gaussian Measures}

In this section, we set out our notation for some standard results about infinite dimensional Gaussian measures which can be found in the following textbooks and lecture notes [4, 9, 25]. Let $\gamma$ be a Gaussian measure on a Hilbert space $(H,\langle\cdot, \cdot\rangle)$. It is characterised by its mean given by the Bochner integral

$$
m=\int_{H} x d \gamma(x)
$$

and the covariance operator $\Gamma: H \rightarrow H$ characterised by the relation

$$
\langle C u, v\rangle=\int\langle u-m, x\rangle\langle v-m, x\rangle d \gamma(x) .
$$

From this it is clear that the covariance operator is positive-definite and self-adjoint. Moreover, we note that $\Gamma$ is necessarily trace-class and the Gaussian can be expressed through eigenvalues $\lambda_{k}^{2}$ and the corresponding eigenbasis $\phi_{k}$

$$
\gamma=\mathcal{L}\left(m+\sum_{i=1}^{\infty} \lambda_{k} \phi_{k} \xi_{k}\right) \text { with } \xi_{k} \stackrel{\text { i.i.d }}{\sim} \mathcal{N}(0,1) .
$$

The Cameron-Martin space associated with $\gamma$ is

$$
H_{\gamma}=\left\{x \mid x=\sum x_{i} \phi_{i} \text { s.t. } \sum \frac{1}{\lambda_{i}^{2}} x_{i}^{2}<\infty\right\} \subset H
$$

equipped with the inner product

$$
\langle x, y\rangle_{\gamma}=\sum \frac{1}{\lambda_{i}^{2}} x_{i} y_{i}
$$

where $x=\sum x_{i} \phi_{i}$ and $y=\sum y_{i} \phi_{i}$. This space characterises the support as well as the direction such that

$$
T_{h \star} \gamma \ll \gamma
$$

where $T_{h}$ is the translation operator $T_{h}(x)=x+h$.

We also consider the Hilbert scale $\left(\mathcal{H}^{s},\|\cdot\|_{s}\right)$ generated by $\gamma$ and the regularity of a draw $\zeta \sim \gamma$ can be expressed as follows.

Lemma Appendix A.2. ([1]) Imposing Assumption 3 the following statements hold:

(i) Let $\zeta$ be a white noise, then $\mathbb{E}\left\|\Gamma^{\frac{\sigma}{2}} \zeta\right\|<\infty$ for all $\sigma>\sigma_{0}$.

(ii) Let $u \sim \mu_{0}$, then $u \in \mathcal{H}^{1-\sigma} \mu_{0}$-a.s. for every $\sigma>\sigma_{0}$. 


\section{Appendix B. Change of Variables for the Posterior}

The state of a model can be described in several ways. In this section, we present the resulting relationship between two different descriptions of the same model.

Theorem Appendix B.1. Suppose $\mathcal{G}_{n}=\mathcal{O}_{n} \circ G$ with $G:\left(X,\|\cdot\|_{X}\right) \rightarrow\left(Y,\|\cdot\|_{Y}\right)$ and $\mathcal{O}:\left(Y,\|\cdot\|_{Y}\right) \rightarrow\left(Z,\|\cdot\|_{Z}\right)$. Furthermore, assume that the posterior $\mu^{y_{n}}\left(\tilde{\mu}^{y}\right)$ is well-defined for the forward operator $\mathcal{G}_{n}\left(\mathcal{O}_{n}\right)$, the prior $\mu_{0}(d a)\left(\tilde{\mu}_{0}(d p)\right)$ and the noise $\xi \sim \mathcal{N}(0, \Gamma)$. It is given by

$$
\begin{aligned}
& \frac{d \mu^{y_{n}}}{d \mu_{0}}(a) \propto \exp \left(-\frac{1}{2}\|\mathcal{G}(a)\|_{\Gamma}^{2}+\langle y, \mathcal{G}(a)\rangle_{\Gamma}\right) \\
& \frac{d \tilde{\mu}^{y}}{d \tilde{\mu}_{0}}(p) \propto \exp \left(-\frac{1}{2}\|\mathcal{O}(p)\|_{\Gamma}^{2}+\langle y, \mathcal{O}(p)\rangle_{\Gamma}\right) .
\end{aligned}
$$

In this case $G_{\star} \mu^{y_{n}}=\tilde{\mu}^{y}$.

Proof. It is sufficient to show that both measures agree on all sets $A \in \mathcal{B}(Y)$

$$
\left(G_{*} \mu^{y_{n}}\right)(A)=\int_{A} 1 d G_{*} \mu^{y_{n}}(d a) .
$$

By the transformation rule

$$
\begin{aligned}
\left(G_{*} \mu^{y_{n}}\right)(A) & =\int_{G^{-1}(A)} 1 d \mu^{y_{n}}(a)=\int_{G^{-1}(A)} c \cdot \exp \left(-\frac{1}{2}\|\mathcal{O}(G(v a))-y\|_{\Gamma}^{2}\right) \mathrm{d} \mu_{0}(a) \\
& =\int_{A} c \cdot \exp \left(-\frac{1}{2}\|\mathcal{O}(v)-y\|_{\Gamma}^{2}\right) d G_{*} \mu_{0}(v) .
\end{aligned}
$$

\section{Appendix C. Proof of Theorem 3.4}

Proof of Theorem 3.4. We follow the same steps as in the proof of Theorem 3.3 up to Equation (17) reading

$$
\left|\left\langle a-a^{\dagger}, \xi\right\rangle_{1}\right| \leq K_{n}\left\|a-a^{\dagger}\right\|_{1}^{\lambda}\left\|a-a^{\dagger}\right\|_{s}^{1-\lambda} 17
$$

with $\lambda=\frac{s-1-\sigma_{0}-\gamma}{s-1}$. We now separate the product using Young's inequality with $\frac{1}{p}+\frac{1}{q}=1$

$$
\begin{aligned}
\left|\left\langle a-a^{\dagger}, \xi\right\rangle_{1}\right| & \leq\left(K_{n}\left(2^{(\lambda-1) q} q n^{-\eta}\right)^{-\frac{1}{q}}\left\|a-a^{\dagger}\right\|_{1}^{\lambda}\right)\left(\left(2^{(\lambda-1) q} q n^{-\eta}\right)^{\frac{1}{q}}\left\|a-a^{\dagger}\right\|_{s}^{1-\lambda}\right) \\
& \leq \tilde{K}_{n}\left\|a-a^{\dagger}\right\|_{1}^{\lambda p}+n^{-\eta}\left(\frac{1}{2}\left\|a-a^{\dagger}\right\|_{s}\right)(1-\lambda) q
\end{aligned}
$$

where, for simplicity of notation, we used $\tilde{K}_{n}:=\frac{K_{n}^{p}\left(2^{-(1-\lambda) q} q n^{-\eta}\right)^{-\frac{p}{q}}}{p}$. 
Lower bound on $\mu^{y_{n}}\left(B_{\epsilon n^{-\kappa}}^{1}\left(a^{\dagger}\right)\right)$ : The following lower bound on $\mu^{y_{n}}\left(B_{\epsilon n^{-\kappa}}^{1}\left(a^{\dagger}\right)\right)$ is based on Equation C.1

$$
\begin{aligned}
\mu^{y_{n}}\left(B_{\epsilon n^{-\kappa}}^{1}\left(a^{\dagger}\right)\right) \geq & \mu^{y_{n}}\left(B_{\frac{\epsilon}{2} n^{-\kappa}}^{1}\left(a^{\dagger}\right) \cap B_{R}^{s}(0)\right) \geq Z(n, \xi) \mu_{0}\left(B_{\frac{\epsilon n-\kappa}{2}}^{1}\left(a^{\dagger}\right) \cap B_{R}^{s}(0)\right) \\
& \cdot \exp \left[-\frac{n^{1-2 \kappa}}{2} \frac{\epsilon^{2}}{4}-n^{\frac{1}{2}-\lambda p \kappa} \tilde{K}_{n}\left(\frac{\epsilon}{2}\right)^{\lambda p}-n^{\frac{1}{2}-\eta}\left[R^{(1-\lambda) q}+\left\|a^{\dagger}\right\|_{s}^{(1-\lambda) q}\right]\right](.
\end{aligned}
$$

The term $n^{1-2 \kappa}$ has to be dominant in Equation (C.2) because the same exponent is appearing in Equation (C.10 except for a larger coefficient. Choosing $R=n^{\theta}$ and substituting the expression for $\tilde{K}_{n}$, this is the case if

$$
\begin{aligned}
1-2 \kappa & >\frac{1}{2}+\eta \frac{p}{q}-\kappa \lambda p \\
1-2 \kappa & >\frac{1}{2}-\eta+(1-\lambda) q \theta \\
\log \mu_{0}\left(B_{\frac{\epsilon n-\kappa}{2}}^{1}\left(a^{\dagger}\right) \cap B_{R}^{s}(0)\right) & \gtrsim n^{1-2 \kappa} .
\end{aligned}
$$

We need small ball probabilities and the exponential moments of $\mu_{0}$ in order to obtain explicit sufficient conditions on $\kappa$. We first note that

$$
\mu_{0}\left(B_{\frac{\epsilon n-\kappa}{2}}^{1}\left(a^{\dagger}\right) \cap B_{R}^{s}(0)\right) \geq \mu_{0}\left(B_{\frac{\epsilon n-\kappa}{2}}^{1}\left(a^{\dagger}\right)\right)-\mu_{0}\left(B_{R}^{s}(0)^{c}\right) .
$$

Equation C.5 holds if

$$
\begin{aligned}
& \rho \kappa<e \theta \\
& \rho \kappa<1-2 \kappa .
\end{aligned}
$$

Upper bound on $\mu^{y_{n}}\left(B_{\epsilon n^{-\kappa}}^{1}\left(a^{\dagger}\right)^{c}\right)$ : We bound $\mu^{y_{n}}\left(B_{\epsilon n^{-\kappa}}^{1}\left(a^{\dagger}\right)^{c}\right)$ by

$$
\mu^{y_{n}}\left(B_{\epsilon n^{-\kappa}}^{1}\left(a^{\dagger}\right)^{c}\right) \leq \mu^{y_{n}}\left(B_{\epsilon n^{-\kappa}}^{1}\left(a^{\dagger}\right)^{c} \cap B_{R}^{s}(0)\right)+\mu^{y_{n}}\left(B_{\epsilon n^{-\kappa}}^{1}\left(a^{\dagger}\right)^{c} \cap B_{R}^{s}(0)^{c}\right) .
$$

Upper bound on $\mu^{y_{n}}\left(B_{\epsilon n^{-\kappa}}^{1}\left(a^{\dagger}\right)^{c} \cap B_{R}^{s}(0)\right)$ : We denote by $M_{B_{\epsilon n^{-\kappa}}^{1}\left(a^{\dagger}\right)^{c} \cap B_{R}^{s}(0)}$ the following supremum

$$
\sup _{B_{\epsilon n^{-\kappa}}^{1}\left(a^{\dagger}\right)^{c} \cap B_{R}^{s}(0)}-\frac{n}{2}\left\|a-a^{\dagger}\right\|_{1}^{2}+\sqrt{n} \tilde{K}_{n}\left\|a-a^{\dagger}\right\|_{1}^{\lambda p}+n^{\frac{1}{2}-\eta}\left(\frac{1}{2}\left\|a-a^{\dagger}\right\|_{s}\right)(1-\lambda) q
$$

which is finite if

$$
\lambda p<2 .
$$

The first two summands above can be rewritten as a function $f$ of $\left\|a-a^{\dagger}\right\|_{1}$ where

$$
f(d)=-\frac{n}{2} d^{2}+\sqrt{n} \tilde{K}_{n} d^{\lambda p} .
$$

By considering $f^{\prime}$, we see that $f$ is decreasing for $d \geq\left(\tilde{K}_{n} \lambda p n^{-\frac{1}{2}}\right)^{\lambda p}$. Thus, for 


$$
\epsilon n^{-\kappa} \geq\left(\tilde{K}_{n} \lambda p n^{-\frac{1}{2}}\right)^{\lambda p}
$$

the following inequality holds

$$
\begin{aligned}
& \mu^{y_{n}}\left(B_{\epsilon n^{-\kappa}}^{1}\left(a^{\dagger}\right)^{c} \cap B_{R}^{s}(0)\right) \\
& \leq Z(n, \xi) \exp \left[-\frac{n^{1-2 \kappa}}{2} \epsilon^{2}+n^{\frac{1}{2}-\lambda p \kappa} \tilde{K}_{n} \epsilon^{\lambda p}+n^{\frac{1}{2}-\eta}\left(R^{(1-\lambda) q}+\left\|a^{\dagger}\right\|_{s}^{(1-\lambda) q}\right)\right] .
\end{aligned}
$$

Then for large $n$, Equation C.9 is implied by

$$
\left(\eta \frac{p}{q}-\frac{1}{2}\right) \lambda p<-\kappa
$$

Upper bound on $\mu^{y_{n}}\left(B_{\epsilon n^{-\kappa}}^{1}\left(a^{\dagger}\right)^{c} \cap B_{R}^{s}(0)^{c}\right)$ : In this section, we bound $\mu^{y_{n}}\left(B_{\epsilon}^{1}\left(a^{\dagger}\right)^{c} \cap\right.$ $\left.B_{R}^{s}(0)^{c}\right)$ using Markov's inequality in combination with the exponential moments of the prior

$$
\begin{aligned}
& \mu^{y_{n}}\left(\exp \left(f\|\cdot\|_{s}^{e}\right) \chi_{B_{\epsilon n}^{1}-\kappa}\left(a^{\dagger}\right)^{c}\right) \leq \int_{B_{\epsilon n^{1} \kappa}^{1}\left(a^{\dagger}\right)^{c}} C(n, \xi) \exp \left(n^{\frac{1}{2}-\eta}\|a\|_{s}(1-\lambda) q\right) \\
& \exp \left(-\frac{n}{2}\left\|a-a^{\dagger}\right\|_{1}^{2}+\sqrt{n} \tilde{K}_{n}\left\|a-a^{\dagger}\right\|_{1}^{\lambda p}+n^{\frac{1}{2}-\eta}\left\|a^{\dagger}\right\|_{s}^{(1-\lambda) q}\right) d \mu_{0}(a) .
\end{aligned}
$$

We denote the term appearing in the exponential in the second line by $T_{0}$. It can be bounded similar to the upper bound on $\mu^{y_{n}}\left(B_{\epsilon n^{-\kappa}}^{1}\left(a^{\dagger}\right)^{c} \cap B_{R}^{s}(0)\right)$

$$
T_{0} \leq \mathfrak{U}_{T_{0}}:=-\frac{n^{1-2 \kappa}}{2} \epsilon^{2}+n^{\frac{1}{2}-\lambda p \kappa} \tilde{K}_{n} \epsilon^{\lambda p}+n^{\frac{1}{2}-\eta}\left\|a^{\dagger}\right\|_{s}^{(1-\lambda) q} .
$$

We denote by $\lesssim$ an inequality with a multiplicative constant not involving $n$ or $\kappa$. In order to get an upper bound for Equation (C.12), we bound the exponential moment by

$$
\mu^{y_{n}}\left(\exp \left(f\|\cdot\|_{s}^{e}\right) \chi_{B_{\epsilon n}^{1}-\kappa}\left(a^{\dagger}\right)^{c}\right) \lesssim C(n, \xi) \int \exp \left(n^{\frac{1}{2}-\eta}\|a\|_{s}^{(1-\lambda) q}+f\|a\|_{s}^{e}+\mathfrak{U}_{T_{0}}\right) d \mu_{0}(d) .
$$

Introducing

$$
\begin{aligned}
& g(r)=n^{\frac{1}{2}-\eta} r^{(1-\lambda) q}+f r^{e} \\
& g^{\prime}(r)=n^{\frac{1}{2}-\eta}(1-\lambda) q r^{(1-\lambda) q-1}+e f r^{e-1}
\end{aligned}
$$

and performing an integration by parts, it follows that

$$
\begin{aligned}
\frac{\mu^{y_{n}}\left(\exp \left(f\|\cdot\|_{s}^{e}\right) \chi_{B^{1}\left(a^{\dagger}\right)^{c}}\right)}{C(n, \xi)} & \lesssim \int \exp \left(g\left(\|a\|_{s}\right)+\mathfrak{U}_{T_{0}}\right) d \mu_{0}(a) \\
& \lesssim \exp \left(\mathfrak{U}_{T_{0}}\right) \int\left[\int_{0}^{\|a\|_{s}} g^{\prime}(r) \exp (g(r)) d r\right]+1 d \mu_{0}(a) \\
& \lesssim \int_{0}^{\infty} g^{\prime}(r) \exp \left(g(r)+\mathfrak{U}_{T_{0}}\right) d \mu_{0}\left(\|a\|_{s}>r\right) d r
\end{aligned}
$$


Posterior Consistency for Bayesian Inverse Problems

$$
\lesssim \int_{0}^{\infty} g^{\prime}(R) \exp \left(n^{\frac{1}{2}-\eta} r^{(1-\lambda) q}-2 f r^{e}\right) d r
$$

The above can only be expected to be finite if

$$
(1-\lambda) q<e .
$$

Moreover, we assume that $\eta<\frac{1}{2}$ since otherwise

$$
\int \exp \left(n^{\frac{1}{2}-\eta}\|a\|_{s}^{(1-\lambda) q}+f\|a\|_{s}^{e}\right) d \mu_{0}(a) \lesssim \int \exp \left(2 f\|a\|_{s}^{e}\right) d \mu_{0}(a) .
$$

In order to achieve an upper bound, we split the term in the exponential into $T_{1}:=$ $n^{\frac{1}{2}-\eta} r^{(1-\lambda) q}-f r^{e}$ and $T_{2}:=-f r^{e}$. The first term is negative whenever

$$
r \geq r_{z}:=\left(n^{\frac{1}{2}-\eta} f^{-1}\right)^{\frac{1}{e-(1-\lambda) q}} .
$$

For $n$ large enough $r_{z} \geq 1$ holds. On the interval $\left[0, s_{z}\right]$ an upper bound $\mathfrak{U}_{T_{1}}$ on the maximum value of $T_{1}$ can be derived as follows

$$
\begin{aligned}
& T_{1}^{\prime}=0 \Rightarrow r=\left((1-\lambda) q n^{\frac{1}{2}-\eta} e^{-1} f^{-1}\right)^{\frac{1}{e-(1-\lambda) q}} \\
& \mathfrak{U}_{T_{1}}:=\left(\frac{(1-\lambda) q}{e f}\right)^{\frac{1}{e-(1-\lambda) q}}\left(n^{\frac{1}{2}-\eta}\right)^{1+\frac{1}{e-(1-\lambda) q}} .
\end{aligned}
$$

Putting everything together gives rise to

$$
\begin{aligned}
\frac{\mu^{y_{n}}\left(\exp \left(f\|\cdot\|_{s}^{e}\right) \chi_{B_{n^{-\kappa}}^{1}\left(a^{\dagger}\right)^{c}}\right)}{C(n, \xi)} & \lesssim \int\left(n^{\frac{1}{2}-\eta}(1-\lambda) q r^{(1-\lambda) q-1}+e f r^{e-1}\right) \exp \left(\mathfrak{U}_{T_{1}}+\mathfrak{U}_{T_{0}}\right) d r \\
& +\int_{r_{z}}^{\infty}\left(n^{\frac{1}{2}-\eta}(1-\lambda) q r^{(1-\lambda) q-1}+e f r^{e-1}\right) \exp \left(\mathfrak{U}_{T_{0}}-f r^{e}\right) d r \\
& \lesssim n^{a} \exp \left(\mathfrak{U}_{T_{1}}+\mathfrak{U}_{T_{0}}\right)
\end{aligned}
$$

for some $a$. Using Markov's inequality, this yields

$$
\mu^{y_{n}}\left(B^{1}\left(a^{\dagger}\right)^{c} \cap B_{R}^{s}(0)^{c}\right) \lesssim C(n, \xi) n^{\frac{1}{2}-\eta} \exp \left(\mathfrak{U}_{T_{0}}+\mathfrak{U}_{T_{1}}-f R^{e}\right)
$$

Again substituting $R=n^{\theta}$, this is asymptotically smaller than $\exp \left(-\frac{n^{1-2 \kappa}}{2} \frac{2}{4}\right)$ if

$$
\left(\frac{1}{2}-\eta\right)\left(1+\frac{1}{e-(1-\lambda) q}\right)<\max (1-2 \kappa, \theta e) .
$$

Collecting the inequalities from above, we see that the results follow by letting $\gamma \rightarrow 0$. 


\section{Appendix D. Normalising Constant of the BRP}

Proof of Lemma 3.2. In order to bound $Z(n, \xi)$ in Equation (12), we rewrite it as

$$
\mu^{y_{n}}=Z(n, \xi) \exp (-\Phi) \mu_{0}
$$

where $Z(n, \xi)=\mu_{0}(\exp (-\Phi))$. We bound $-\Phi$ using the Cauchy-Schwarz inequality

$$
-\Phi \leq-\frac{1}{2} n\|a\|_{1}^{2}+n\left\|a^{\dagger}\right\|_{1}\|a\|_{1}+n^{\frac{1}{2}}\langle a, \xi\rangle_{1} .
$$

The following steps are quite similar to the steps in the proof of the Theorems 3.3 and 3.4. We treat $\langle a, \xi\rangle_{1}$ by smoothing $\xi$ at the expense of $a$

$$
\begin{aligned}
\left|\langle a, \xi\rangle_{1}\right| & \leq\left|\left\langle\Gamma^{-1+\frac{1-\sigma_{0}-\gamma}{2}} a, \Gamma^{\frac{\sigma_{0}-1+\gamma}{2}} \xi\right\rangle\right| \\
& \leq\|a\|_{1+\sigma_{0}+\gamma}\|\xi\|_{1-\sigma_{0}-\gamma} .
\end{aligned}
$$

We use the interpolation inequality for Hilbert scales with $\lambda=\frac{s-1-\sigma_{0}-\gamma}{s-1}$ (see Lemma Appendix A.1p and Hölder's inequality with $\frac{1}{p}+\frac{1}{q}=1$ to obtain

$$
\|a\|_{1+\sigma_{0}+\gamma} \leq\|a\|_{1}^{\lambda}\|a\|_{s}^{1-\lambda} \leq \frac{\|a\|_{1}^{p \lambda}}{p}+\frac{\|a\|_{1}^{q(1-\lambda)}}{q} .
$$

Combining these bounds yields

$$
-\Phi \leq-\frac{1}{2} n\|a\|_{1}^{2}+n\left\|a^{\dagger}\right\|_{1}\|a\|_{1}+\frac{\|a\|_{1}^{p \lambda}}{p}\|\xi\|_{1-\sigma_{0}-\gamma}+\frac{\|a\|_{1}^{q(1-\lambda)}}{q}\|\xi\|_{1-\sigma_{0}-\gamma} .
$$

The first three terms are bounded in $a$ because they are dominated by the first if $\lambda p<2$. This is implied by choosing $q=\frac{2+\gamma}{2-\lambda}$. Note that $\|\xi\|_{1-\sigma_{0}-\gamma}$ is $\mu_{\xi_{n}}$-a.s. bounded due to Lemma Appendix A.2. Thus $Z(n, q)$ is bounded below if $e>q$. Letting $\gamma \downarrow 0$ in $q$ we see that this is the case for

$$
e>\frac{2 \sigma_{0}}{s-1+\sigma_{0}} \text {. }
$$

An upper bound on $Z(n, q)$ follows from a simple lower bound on $-\Phi$ on $B_{M}^{1+\sigma+\gamma}(0)$ and the prior measure of this set.

[1] S. Agapiou, S. Larsson, and A. M. Stuart. Posterior Consistency of the Bayesian Approach to Linear Ill-Posed Inverse Problems. Stochastic Processes and Applications, 2013. to appear.

[2] S. Agapiou, A. M. Stuart, and Y.-X. Zhang. Bayesian Posterior Contraction Rates for Linear Severely Ill-posed Inverse Problems. ArXiv e-prints, October 2012.

[3] G. Alessandrini. Stable determination of conductivity by boundary measurements. Appl. Anal., 27(1-3):153-172, 1988.

[4] Vladimir I. Bogachev. Gaussian measures, volume 62 of Mathematical Surveys and Monographs. American Mathematical Society, Providence, RI, 1998.

[5] C. Borell. Convex measures on locally convex spaces. Ark. Mat., 12:239-252, 1974.

[6] T. Choi and M. J. Schervish. On posterior consistency in nonparametric regression problems. J. Multivariate Anal., 98(10):1969-1987, 2007.

[7] A. Cohen, R. A. Devore, and C. Schwab. Convergence rates of best $N$-term Galerkin approximations for a class of elliptic sPDEs. Found. Comput. Math., 10(6):615-646, 2010. 
[8] A. Cohen, R. A. Devore, and C. Schwab. Analytic regularity and polynomial approximation of parametric and stochastic elliptic PDE's. Anal. Appl. (Singap.), 9(1):11-47, 2011.

[9] Giuseppe Da Prato and Jerzy Zabczyk. Stochastic equations in infinite dimensions, volume 44 of Encyclopedia of Mathematics and its Applications. Cambridge University Press, Cambridge, 1992.

[10] M. Dashti, S. Harris, and A. M Stuart. Besov priors for Bayesian inverse problems. Inverse Probl. Imaging, 6:183-200, 2012.

[11] M. Dashti, K. J. H. Law, A. M. Stuart, and J. Voss. MAP Estimators and Posterior Consistency in Bayesian Nonparametric Inverse Problems. ArXiv preprint 1303.4795, 2013.

[12] M. Dashti and A. M. Stuart. Uncertainty quantification and weak approximation of an elliptic inverse problem. SIAM J. Numer. Anal., 49:2524-2542, 2011.

[13] R. A. DeVore. Nonlinear approximation. In Acta numerica, 1998, volume 7 of Acta Numer., pages 51-150. Cambridge Univ. Press, Cambridge, 1998.

[14] P. Diaconis and D. A. Freedman. On inconsistent Bayes estimates of location. Ann. Statist., 14(1):68-87, 1986.

[15] P. Diaconis and D. A. Freedman. On the consistency of Bayes estimates. Ann. Statist., 14(1):1-67, 1986. With a discussion and a rejoinder by the authors.

[16] J. L. Doob. Application of the theory of martingales. Le calcul des probabilites et ses applications, pages 23-27, 1949.

[17] Heinz W. Engl, Martin Hanke, and Andreas Neubauer. Regularization of inverse problems, volume 375 of Mathematics and its Applications. Kluwer Academic Publishers Group, Dordrecht, 1996.

[18] Lawrence C. Evans. Partial differential equations, volume 19 of Graduate Studies in Mathematics. American Mathematical Society, Providence, RI, second edition, 2010.

[19] Frédéric Ferraty and Philippe Vieu. Nonparametric functional data analysis. Springer Series in Statistics. Springer, New York, 2006. Theory and practice.

[20] J.-P. Florens and A. Simoni. Regularized posteriors in linear ill-posed inverse problems. Scand. J. Stat., 2012.

[21] D. A. Freedman. On the asymptotic behavior of Bayes' estimates in the discrete case. Ann. Math. Statist., 34:1386-1403, 1963.

[22] S. Ghosal, J. K. Ghosh, and A. W. van der Vaart. Convergence rates of posterior distributions. Ann. Statist., 28(2):500-531, 2000.

[23] Subhashis Ghosal and Aad van der Vaart. Fundamentals of nonparametric bayesian inference. 2012.

[24] David Gilbarg and Neil S. Trudinger. Elliptic partial differential equations of second order. Classics in Mathematics. Springer-Verlag, Berlin, 2001. Reprint of the 1998 edition.

[25] M. Hairer. An Introduction to Stochastic PDEs. Lecture Notes, 2009.

[26] M. Hairer, A. M. Stuart, and J. Voss. Analysis of SPDEs Arising in Path Sampling. Part 2: The Nonlinear Case. Ann. Appl. Probab., pages 1657-1706, 2007.

[27] T. M. Hansen, K. S. Cordua, and K. Mosegaard. Inverse problems with non-trivial priors: Efficient solution through sequential Gibbs sampling. Comput. Geosci., pages 1-19, 2012.

[28] Nils Lid Hjort, Chris Holmes, Peter Müller, and Stephen G. Walker, editors. Bayesian nonparametrics, volume 28 of Cambridge Series in Statistical and Probabilistic Mathematics. Cambridge University Press, Cambridge, 2010.

[29] V. H. Hoang, C. Schwab, and A. M. Stuart. Sparse MCMC gpc Finite Element Methods for Bayesian Inverse Problems. ArXiv preprint 1207.2411, July 2012.

[30] B. T. Knapik, A. W. van der Vaart, and J. H. van Zanten. Bayesian inverse problems with Gaussian priors. Ann. Statist., 39(5):2626-2657, 2011.

[31] P. Kuchment and G. Uhlmann. The Radon and X-Ray Transforms. 2012.

[32] W. V. Li and Q.-M. Shao. Gaussian processes: inequalities, small ball probabilities and applications. In Stochastic processes: theory and methods, volume 19 of Handbook of Statist., pages 533-597. North-Holland, Amsterdam, 2001. 
[33] M. A. Lifshits. Gaussian random functions, volume 322 of Mathematics and its Applications. Kluwer Academic Publishers, Dordrecht, 1995.

[34] M. A. Lifshits. Bibliography of small deviation probabilities, July 2012.

[35] K. Ray. Bayesian inverse problems with non-conjugate priors. arXiv preprint arXiv:1209.6156, 2012.

[36] G. R. Richter. An inverse problem for the steady state diffusion equation. SIAM J. Appl. Math., 41(2):210-221, 1981.

[37] John Roe. Elliptic operators, topology and asymptotic methods, volume 395 of Pitman Research Notes in Mathematics Series. Longman, Harlow, second edition, 1998.

[38] C. Schwab and A. M. Stuart. Sparse deterministic approximation of Bayesian inverse problems. Inverse Probl., 28(4):045003, 32, 2012.

[39] X. Shen and L. Wasserman. Rates of convergence of posterior distributions. Ann. Statist., 29(3):687-714, 2001.

[40] A. M. Stuart. Inverse problems: a Bayesian perspective. Acta Numer., 19:451-559, 2010.

[41] A. M. Stuart. The Bayesian Approach To Inverse Problems. ArXiv preprint 1302.6989, 2013.

[42] Hans Triebel. Function spaces and wavelets on domains, volume 7 of EMS Tracts in Mathematics. European Mathematical Society (EMS), Zürich, 2008.

[43] A. W. van der Vaart and J. H. van Zanten. Rates of contraction of posterior distributions based on Gaussian process priors. Ann. Statist., 36(3):1435-1463, 2008.

[44] S. Walker. New approaches to Bayesian consistency. Ann. Statist., 32(5):2028-2043, 2004.

[45] H. Weyl. Das asymptotische Verteilungsgesetz der Eigenwerte linearer partieller Differentialgleichungen (mit einer Anwendung auf die Theorie der Hohlraumstrahlung). Math. Ann., 71(4):441-479, 1912.

[46] William WG Yeh. Review of parameter identification procedures in groundwater hydrology: The inverse problem. Water Resources Research, 22(2):95-108, 1986. 